\title{
Bulk-edge and bulk-hinge correspondence in inversion-symmetric insulators
}

\author{
Ryo Takahashi, ${ }^{1}$ Yutaro Tanaka $\odot,{ }^{1}$ and Shuichi Murakami ${ }^{1,2}$ \\ ${ }^{1}$ Department of Physics, Tokyo Institute of Technology, 2-12-1 Ookayama, Meguro-ku, Tokyo 152-8551, Japan \\ ${ }^{2}$ TIES, Tokyo Institute of Technology, 2-12-1 Ookayama, Meguro-ku, Tokyo 152-8551, Japan
}

(Received 21 October 2019; accepted 12 February 2020; published 12 March 2020)

\begin{abstract}
We show that a slab of a three-dimensional inversion-symmetric higher-order topological insulator (HOTI) in class $\mathrm{A}$ is a $2 \mathrm{D}$ Chern insulator, and that in class AII is a $2 \mathrm{D} Z_{2}$ topological insulator. We prove it by considering a process of cutting the three-dimensional inversion-symmetric HOTI along a plane and study the spectral flow in the cutting process. We show that the $Z_{4}$ indicators, which characterize three-dimensional inversion-symmetric HOTIs in classes A and AII, are directly related to the $Z_{2}$ indicators for the corresponding two-dimensional slabs with inversion symmetry, i.e., the Chern number parity and the $Z_{2}$ topological invariant, for classes A and AII, respectively. The existence of the gapless hinge states is understood from the conventional bulk-edge correspondence between the slab system and its edge states. Moreover, we also show that the spectral-flow analysis leads to another proof of the bulk-edge correspondence in one- and two-dimensional inversion-symmetric insulators.
\end{abstract}

DOI: 10.1103/PhysRevResearch.2.013300

\section{INTRODUCTION}

Bulk-edge correspondence is one of the key concepts in the field of a topological insulator (TI) [1,2], which associates the nontrivial topology of the bulk wave functions with existence of anomalous surface states. In the quantum Hall insulator (QHI), which is characterized by the bulk Chern number [3], chiral edge modes appear at the edges of the system [4,5]. In the quantum spin Hall insulator [6-8], which is the timereversal-symmetric counterpart of the QHI and characterized by the $Z_{2}$ topological invariant, helical edge modes appear at the edges of the system.

In inversion-symmetric insulators, inversion parities at time-reversal-invariant momenta (TRIM) are topological invariants. When they take nontrivial values, the insulator shows unusual properties at the edges. In one-dimensional centrosymmetric insulators, the Zak phase is quantized, and its value is evaluated from the inversion parities [9]. As the Zak phase is proportional to polarization, in the system with the nonzero Zak phase, fractional surface charges appear at the end of the system [10-12]. In two-dimensional centrosymmetric insulators, the Chern number parity is evaluated from the inversion parities [13-16]. When the Chern number parity is odd, an odd number of chiral edge modes appear at the edges of the system.

In recent years, a new class of a TI, called higher-order topological insulator (HOTI) [17-47], has been proposed. A three-dimensional HOTI is insulating both in the bulk and in the surface. However, it has one-dimensional anomalous gapless states at the hinges, which are intersections of two

Published by the American Physical Society under the terms of the Creative Commons Attribution 4.0 International license. Further distribution of this work must maintain attribution to the author(s) and the published article's title, journal citation, and DOI. surfaces. The correspondence between the bulk topology and the anomalous gapless states at the hinges is called bulk-hinge correspondence, which is an extension of the conventional bulk-edge correspondence.

To the best of our knowledge, in the previous studies, explanations of bulk-hinge correspondence are roughly classified into two approaches: (i) $\boldsymbol{k} \cdot \boldsymbol{p}$ theory approach [23-25,32$34,36,38,42,43]$, and (ii) Wannier approach $[25,26,37,42]$. In approach (i), one starts from the surface Dirac Hamiltonian, which represents anomalous gapless surface states as a lowenergy effective Hamiltonian for the surface. By adding a symmetry-respecting mass term, the surface energy spectrum becomes gapped. However, on the surface of the HOTI, the sign of the mass term depends on the surface direction. Therefore, at the hinge, shared by two surfaces with opposite signs of the mass terms, the mass term is zero. It means that the hinge states remain gapless. In approach (ii), we consider the two two-dimensional high-symmetry subspaces (e.g., $k_{z}=0$ and $k_{z}=\pi$ ) in the three-dimensional $\boldsymbol{k}$ space as pseudo-twodimensional systems, and we calculate the Wannier centers (WCs) for the states in these subspaces. If excess corner charges at the two subspaces calculated from the WCs are different, then there exist hinge states which compensate for the difference of the corner charges.

Both approaches have some drawbacks. Both of them are proofs only for special models, and it is not clear whether they can be applied to general systems. First, the $\boldsymbol{k} \cdot \boldsymbol{p}$ theory approach cannot be applied to systems whose surfaces are not described by the Dirac model. Therefore, one cannot conclude existence of hinge states for general systems from this argument. Second, in the WC approach, the exact value of the excess corner charge is concluded only in the case of completely localized Wannier states, which have no hopping terms. Therefore, to complete the proof, it is necessary to relate the value of the excess corner charge with topological invariants for general systems. 
In this paper, we introduce another approach, which is applicable to more general tight-binding models with inversion symmetry. We introduce an open boundary to a 3D inversion-symmetric HOTI via cutting procedure [48]. In this process, the boundary condition continuously changes from the periodic one to the open one, while maintaining inversion symmetry. By examining the spectral flow in the cutting process, we obtain restrictions on the inversion parities of the resulting 2D slab system. Thus, in this paper, we show that the $Z_{4}$ indicator $\mu_{1}$, which characterizes 3D inversion-symmetric HOTIs in class A, is directly related to the Chern number parity of the resulting 2D slab system in class A. Likewise, we also show that the $Z_{4}$ indicator $\kappa_{1}$, which characterizes $3 \mathrm{D}$ inversion-symmetric HOTIs in class AII, is directly related to the $Z_{2}$ topological invariant of the resulting $2 \mathrm{D}$ slab system in class AII. If we cut the 3D HOTI via the cutting procedure, then the corresponding 2D topological invariant of the resulting 2D system always takes a nonzero value, as we show in this paper. Due to the conventional bulk-edge correspondence, the resulting $2 \mathrm{D}$ system has an odd number of anomalous edge modes at the edges. Since the edges of the resulting 2D system are nothing but the hinges of the original 3D system, this gives a proof of the bulk-hinge correspondence.

Moreover, we apply the same approach to one- and twodimensional inversion-symmetric topological insulators. As a result, we obtain another proof of bulk-edge correspondence. In the previous papers, inversion parities are related to bulk topological invariants, such as the Zak phase and the Chern number parity. In our approach, inversion parities are directly related to unusual surface properties, such as the fractional end charge and the chiral edge modes.

The expectation that the HOTI slab is equivalent to the 2D Chern insulator is discussed in some previous works [40,41]. However, the discussions in these works are based on specific models, and it has been unclear whether any HOTI slab is a Chern insulator or not. In this paper, we clarify that the expectation is correct for any inversion-symmetric HOTI, and find the formula, Eq. (18), without using specific models.

We note that, in this paper, we consider a process of cutting the three-dimensional HOTI along a plane. In another paper, we consider a process of cutting the three-dimensional HOTI along two planes [49]. In the paper, we discuss allowed positions of the hinge states, which is determined only if we consider a cutting along two planes. Thus, different cutting procedure reveal different aspects of hinge states.

This paper is organized as follows. In Sec. II, we introduce the cutting procedure and study the spectral flow in the cutting process. As a result, we obtain another proof of bulk-edge correspondence in a 1D inversion-symmetric topological insulator. In Sec. III, by using the result of Sec. II, we show another proof of the famous relation between the number of chiral edge modes and the inversion parities at time-reversalinvariant momenta (TRIM). In Sec. IV, we show that $\mu_{1}$, the indicator of 3D inversion-symmetric HOTI, is directly connected to the 2D indicator, i.e., Chern number parity. In Sec. V, we generalize the results of Secs. II-IV to timereversal symmetric systems, i.e., class AII systems, and show that $\kappa_{1}$ is directly connected to the $Z_{2}$ topological invariant. In Sec. VI, we discuss some exceptions to the previous sections and the bulk-boundary correspondence in them. Conclusion are given in Sec. VII.

\section{BULK-EDGE CORRESPONDENCE IN 1D CLASS A}

We start with a noninteracting centrosymmetric system on a one-dimensional lattice with periodic boundary conditions. In such a system, $n_{ \pm}\left(\Gamma_{j}\right)$, the number of occupied states with even (+) and odd (-) parities at a TRIM $\Gamma_{j}$ are topological invariants. Particularly for insulators, their sum is equal to the number of occupied bands $v$, i.e., $n_{+}\left(\Gamma_{j}\right)+$ $n_{-}\left(\Gamma_{j}\right)=v\left({ }^{\forall} \Gamma_{j}\right)$. In one-dimensional systems, there are two TRIM, $k_{1}=0, \pi$, where $k_{1}$ is the momentum. Therefore, in an inversion-symmetric insulator, we have three independent topological numbers $\left[v, n_{-}(0), n_{-}(\pi)\right]$. If one of these numbers is different between two insulators, then they are topologically different in that they cannot be continuously deformed to each other without gap closing or breaking inversion symmetry.

Here, we briefly discuss a choice of the unit cell and the inversion center. We take the unit cell to be invariant under the inversion operation. Then, there are two inequivalent inversion centers for the unit cell; one is at the center of the unit cell and the other is at the boundary of the unit cell, and they are displaced by a half of the primitive lattice vector. In this section, we assume that the number of unit cells, $L$, is odd, and we choose the inversion center to be the center of the unit cell. As we explain in Appendix B, when $L$ is even, we should change the choice of the inversion center. We also note that this unit-cell choice cannot be applied to some models, as we discuss in Sec. VI. In such models, the conventional bulk-edge correspondence shown in this section does not work, and we show that a modified bulk-edge correspondence holds in these cases [46].

In this section, we show that for a centrosymmetric onedimensional system with an open boundary condition, when $n_{-}(0)-n_{-}(\pi)=1(\bmod 2)$, the number of occupied states $\left(N_{\mathrm{OBC}}\right)$ have a different parity from that with periodic boundary condition $\left(N_{\mathrm{PBC}}\right)$, i.e., $N_{\mathrm{OBC}}=N_{\mathrm{PBC}}+1(\bmod 2)$. More precisely, we show the following relation:

$$
N_{\mathrm{OBC}}=N_{\mathrm{PBC}}+n_{-}(0)-n_{-}(\pi) \quad(\bmod 2) .
$$

As we will mention later, this result is closely related to a fractional end charge. This is the conventional bulk-edge correspondence in 1D inversion-symmetric insulators.

\section{A. General proof}

Here, we assume that no atomic site is located at the unit-cell boundary. We set the system size to be $L|\boldsymbol{a}|$, where $\boldsymbol{a}$ is the primitive lattice vector. For simplicity, let $L$ be an odd integer, that is, $L=2 M+1$ with an integer $M$. The case of even $L$ is discussed in Appendix B. For each unit cell, we associate a lattice site with the middle of the unit cell. Let $x_{1}$ be the coordinate along the 1D system, with $x_{1}=-M,-M+1, \ldots, M-1, M$ being the lattice sites for the tight-binding model. We first begin with a periodic chain, by connecting between the unit cells at the two sites $x= \pm M$. Next, in the following way, we introduce a cutting procedure, which is used in the Appendix of Ref. [48] for class AII systems. We replace the hopping amplitudes $t_{j}$ for all the bonds that cross the boundary between $x_{1}=-M$ and $x_{1}=M$ by $\lambda t_{j}$, where $\lambda$ is real. We note that if $\lambda=1$, then the 

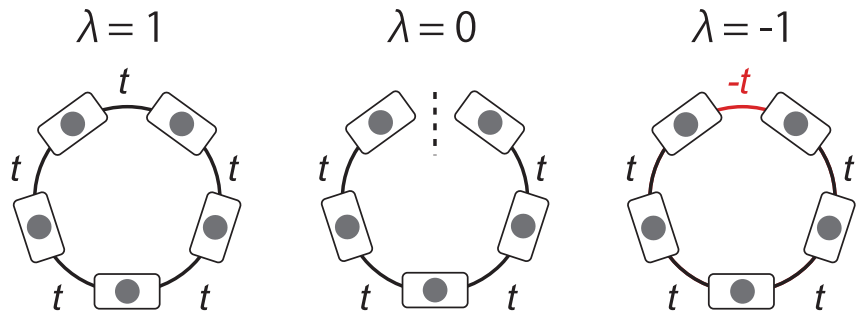

FIG. 1. Conceptual figure of a one-dimensional inversionsymmetric insulator cut at the boundary. When $\lambda=1$, the system is periodic, and when $\lambda=0$, the system is open. When $\lambda=-1$, the system is antiperiodic.

system is periodic in $x_{1}$, and if $\lambda=0$, then the system is open in the $x_{1}$ direction. Figure 1 is a conceptual figure of the one-dimensional inversion-symmetric insulator cut at the boundary. For any real values of $\lambda$, inversion symmetry is always preserved. Let $N_{-(+)}$be the number of occupied states with odd (even) parity; these values depend on $\lambda$. First of all, we show that the value of $N_{-}$is evaluated from $n_{-}\left(\Gamma_{j}\right)$ for some specific values of $\lambda$.

First, we consider the case of $\lambda=1$, when the system is periodic in $x_{1}$. In this case, the Bloch wave number $k_{1}$ takes the following values:

$$
k_{1}=\frac{2 \pi}{L} m_{1} \quad\left(-M \leqslant m_{1} \leqslant M, M: \text { integer }\right) .
$$

Because $L(=2 M+1)$ is an odd number, $k_{1}$ is a TRIM if and only if $k_{1}=0$, as shown in Fig. 2(a). For other values of $k_{1}=k_{*}(\neq 0)$, the inversion operation changes $k_{*}$ to $-k_{*}$. One can construct eigenstates of the inversion operator $\mathcal{P}$ with eigenvalues +1 and -1 from $\psi_{m}\left(k_{*}\right)$ and $\psi_{m}\left(-k_{*}\right)$ as $\frac{1}{\sqrt{2}}\left[\psi_{m}\left(k_{*}\right) \pm \mathcal{P} \psi_{m}\left(k_{*}\right)\right]$, where $\psi_{m}(k)$ is a Bloch eigenstate and $\mathcal{P} \psi_{m}\left(k_{*}\right) \propto \psi_{m}\left(-k_{*}\right)$. Therefore, each pair $\left(k_{*},-k_{*}\right)$ contributes 1 to $N_{-}$. For an insulating system, the total number of non-TRIM pairs is evaluated as $(L-1) v / 2$, where $v$ is the number of occupied bands. Therefore, when $\lambda=1, N_{-}$can be expressed as follows:

$$
\left.N_{-}\right|_{\lambda=1}=\frac{(L-1) v}{2}+n_{-}(0)
$$

Next, we consider the case of $\lambda=-1$, when the system is antiperiodic in $x_{1}$. In this case, by performing a unitary transformation $U_{1}=\exp \left[i \pi \hat{x_{1}} / L\right]$, where $\hat{x_{1}}$ is the position operator, the periodicity in the $x_{1}$ direction can be restored,
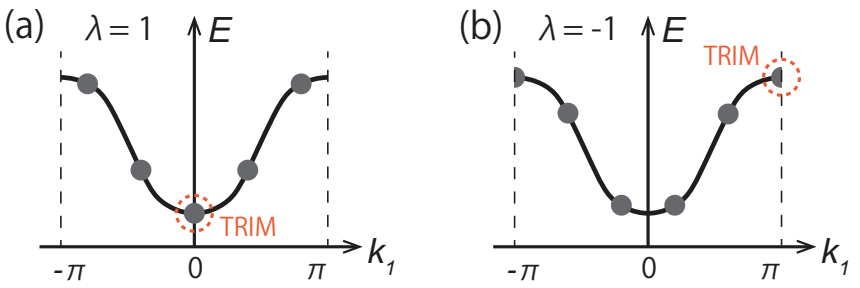

FIG. 2. Two examples of the bulk energy levels at $\lambda= \pm 1$. All of the states except for those at TRIM are paired by inversion operation. (a) For $\lambda=+1$, the state at $k_{1}=0$ is not paired. (b) For $\lambda=-1$, the state at $k_{1}=\pi$ is not paired. but the Bloch wave vector is shifted as $k_{1} \rightarrow k_{1}+\pi / L$ (see Appendix A). Then $k_{1}$ takes the following values:

$$
k_{1}=\frac{2 \pi}{L} m_{1}+\frac{\pi}{L} \quad\left(-M \leqslant m_{1} \leqslant M\right) .
$$

Now, as shown in Fig. 2(b), $k_{1}$ is a TRIM if and only if $k_{1}=\pi$. Therefore,

$$
\left.N_{-}\right|_{\lambda=-1}=\frac{(L-1) v}{2}+n_{-}(\pi) .
$$

Next, we follow the change in $N_{-}$through the change of $\lambda$ from 1 to -1 . From Eqs. (3) and (5), the total change in $N_{-}$is evaluated from $n_{-}$:

$$
\left.N_{-}\right|_{\lambda=1}-\left.N_{-}\right|_{\lambda=-1}=n_{-}(0)-n_{-}(\pi) .
$$

We consider the energy spectrum in the process of changing $\lambda$. In this process, the Hamiltonian changes only at the boundary. Therefore, only the boundary localized states have strong $\lambda$ dependence. We can show that, for sufficiently large $L$, the energy spectrum is symmetric with respect to the transformation $\lambda \leftrightarrow-\lambda$, and the bound states $\left|\psi_{l}(\lambda)\right\rangle$ and $\left|\psi_{l}(-\lambda)\right\rangle$ have opposite parities (see Appendix A2).

This is roughly understood as follows. We assume that the effective Hamiltonian of the bound states are roughly expressed in the basis of the localized states at the two ends $\left(\psi_{L}, \psi_{R}\right)$

$$
\mathcal{H}_{\text {eff }} \simeq\left(\begin{array}{cc}
0 & \lambda t \\
\lambda t & 0
\end{array}\right) .
$$

Here, for simplicity, we consider the hopping $\lambda t$ between two ends is real. The bound states are expressed as bonding and antibonding states between $\psi_{L}$ and $\psi_{R}\left(=\mathcal{P} \psi_{L}\right)$. The bound states $\frac{1}{\sqrt{2}}\left(\psi_{L} \pm \psi_{R}\right)$ have energies $E= \pm \lambda t$, and they have inversion parities $p= \pm 1$ (double sign in the same order). Therefore, when we transform $\lambda$ as $\lambda \leftrightarrow-\lambda$, the bound states change the signs of their energies. If we compare the states between $\lambda=\lambda_{*}$ and $\lambda=-\lambda_{*}$ for an arbitrary values of $\lambda_{*}$, then the bound states have the same energy, but opposite signs of the inversion parity. As seen from this example, roughly speaking, the transformation $\lambda \leftrightarrow-\lambda$ inverts the energy levels of bonding and antibonding states at the boundary. Its detailed proof is shown in Appendix A2.

From the property under the transformation $\lambda \leftrightarrow-\lambda$, the following relation holds (double sign in the same order):

$$
\left[N_{ \pm}\right]_{\lambda=0}^{\lambda=1}=\left[N_{\mp}\right]_{\lambda=0}^{\lambda=-1} .
$$

Here, we used the following notation: $\left.[X]_{\lambda=a}^{\lambda=b} \stackrel{\text { def }}{=} X\right|_{\lambda=b}-$ $\left.X\right|_{\lambda=a}$. By using Eqs. (6) and (8), the difference between $\left.N_{\mathrm{PBC}} \equiv N\right|_{\lambda=1}$ and $\left.N_{\mathrm{OBC}} \equiv N\right|_{\lambda=0}$ is evaluated as follows:

$$
\begin{aligned}
{[N]_{\lambda=0}^{\lambda=1} } & =\left[N_{+}\right]_{\lambda=0}^{\lambda=1}+\left[N_{-}\right]_{\lambda=0}^{\lambda=1} \\
& =\left[N_{-}\right]_{\lambda=0}^{\lambda=-1}+\left[N_{-}\right]_{\lambda=0}^{\lambda=1} \\
& =\left[N_{-}\right]_{\lambda=1}^{\lambda=-1}+2\left[N_{-}\right]_{\lambda=0}^{\lambda=1}
\end{aligned}
$$

Therefore, we get

$$
\left.N\right|_{\lambda=0}=\left.N\right|_{\lambda=1}+n_{-}(0)-n_{-}(\pi)+2\left[N_{-}\right]_{\lambda=1}^{\lambda=0} .
$$

Equation (1) is derived by taking modulo 2 on both sides of the Eq. (10). 

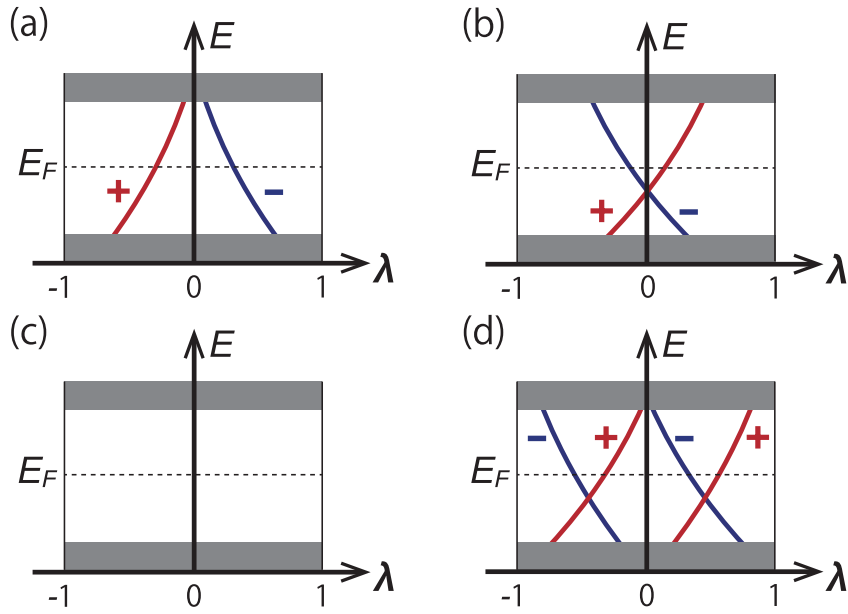

FIG. 3. Four representative examples of the spectral flow for the change of $\lambda$. Red and blue lines, which are marked with + and - , represent bound states with even and odd parity, respectively. The shaded areas represent the bulk energy spectra. The number of occupied states with odd parity at TRIM, $\left[n_{-}(0), n_{-}(\pi)\right]$, is (a), (b) $(1,0),(\mathrm{c})(0,0)$, and (d) $(2,0)$. In (a), (b), $N_{\mathrm{OBC}}$, the number of states below the Fermi energy $E_{F}$ at $\lambda=0$, is one less or more than that of the bulk, $N_{\mathrm{PBC}}$. In (c), (d), $N_{\mathrm{OBC}}$ and $N_{\mathrm{PBC}}$ are equal.

Here, we explain the relation of this result and the fractional end charge. From Eq. (1), when $n_{-}(0)-n_{-}(\pi) \equiv 1$ $(\bmod 2)$, through the change from $\lambda=1$ to $\lambda=0$, the number of occupied states changes by an odd number. Since the Hamiltonian is changed only at the boundary, newly occupied states are localized at the boundary, and it causes total excess charges $(2 m+1) e(m$ : integer) at the two ends of the system. If we assume that the system preserves inversion symmetry, then the excess charges should be divided equally to the two ends. Therefore, each end have a fractional excess charge $\left(m+\frac{1}{2}\right) e$.

\section{B. Illustrative examples}

Here, we illustrate how the spectrum changes through the change of $\lambda$ for three examples: (i) $\left[n_{-}(0), n_{-}(\pi)\right]=(1,0)$, (ii) $(0,0)$, and (iii) $(2,0)$. By considering the spectral flow, we can visualize how Eq. (10) is satisfied. In the case (i), when $\lambda$ is changed from +1 to -1 , one state with odd parity crosses the Fermi energy $E_{F}$ from below, and one state with even parity crosses $E_{F}$ from above. Furthermore, the energy spectrum is symmetric with respect to the transformation $\lambda \leftrightarrow$ $-\lambda$, and the bound states $\left|\psi_{l}(\lambda)\right\rangle$ and $\left|\psi_{l}(-\lambda)\right\rangle$ have opposite parities (see Appendix A2). Figures 3(a) and 3(b) show two representative examples of the energy spectra which satisfy these requirements. In Fig. 3(a), the number of occupied states is one less than that of the bulk, i.e., $N_{\mathrm{OBC}}=N_{\mathrm{PBC}}-1$. In Fig. 3(b), the number of occupied states is one more than that of the bulk, i.e., $N_{\mathrm{OBC}}=N_{\mathrm{PBC}}+1$. In both cases $N_{\mathrm{OBC}}$ have different parity from that of $N_{\mathrm{PBC}}$. In the case (ii) $\left[n_{-}(0), n_{-}(\pi)\right]=(0,0)$, the total change in $N_{-}$is equal to 0. Figure 3(c) shows an example of the energy spectrum. In Fig. 3(c), there is no state which crosses $E_{F}$, and $N_{\mathrm{OBC}}=$ $N_{\mathrm{PBC}}$. Finally, we consider case (iii) $\left[n_{-}(0), n_{-}(\pi)\right]=(2,0)$. In this case, when $\lambda$ is changed from +1 to -1 , two odd-
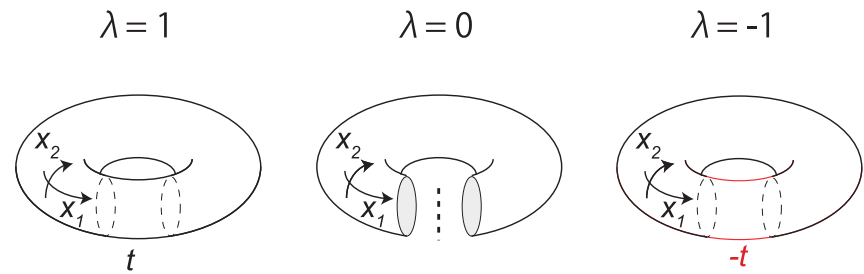

FIG. 4. Conceptual figure of a two-dimensional inversionsymmetric insulator cut at the boundary along the $x_{1}$ direction. When $\lambda=1$, the system is periodic, and when $\lambda=0$, the system is an open finite system at $x_{1}=$ const. When $\lambda=-1$, this system is antiperiodic along the $x_{1}$ direction.

(even-) parity states cross $E_{F}$ from below (above). One of the examples is shown in Fig. 3(d), and $N_{\mathrm{OBC}}$ is equal to that of the bulk. In both cases (ii) and (iii), $N_{\mathrm{OBC}}$ have the same parity with that of $N_{\mathrm{PBC}}$.

\section{BULK-EDGE CORRESPONDENCE IN 2D CLASS A}

In this section, we show bulk-edge correspondence in two-dimensional inversion-symmetric insulators. We consider a noninteracting centrosymmetric system on a twodimensional lattice with periodic boundary conditions. In a two-dimensional system, there are four TRIM, $\left(k_{1}, k_{2}\right)=$ $(0,0),(\pi, 0),(0, \pi),(\pi, \pi)$, which we label with $\Gamma, X, Y, M$, respectively. Therefore, in an insulator, we have five independent topological numbers $\left[v, n_{-}\left(\Gamma_{j}\right)\right]$, where $\Gamma_{j}$ represent the TRIM.

In this section, we show that for an insulating system with an open boundary condition, when $\sum_{\Gamma_{j}} n_{-}\left(\Gamma_{j}\right)=1(\bmod 2)$, there are an odd number of chiral edge modes at the boundary. A similar result has been already shown in previous works [13-16] in the form

$$
\prod_{\Gamma_{j} \in \operatorname{TRIM}}(-1)^{n_{-}\left(\Gamma_{j}\right)}=(-1)^{\mathrm{Ch}},
$$

where $\mathrm{Ch}$ is the Chern number of the system. Since the Chern number is equal to the number of chiral edge modes [4,5], this equation also means the existence of chiral edge modes when $\sum_{\Gamma_{j}} n_{-}\left(\Gamma_{j}\right)=1(\bmod 2)$. In this sense, we give another proof of this equation.

\section{A. General proof}

We consider a two-dimensional crystal with primitive vectors $\boldsymbol{a}_{i}(i=1,2)$. Let $L_{i}(i=1,2)$ be the length of the system along $\boldsymbol{a}_{i}$, measured in units of $\left|\boldsymbol{a}_{i}\right|(i=1,2)$. For simplicity, let $L_{1}$ be an odd integer, $L_{1}=2 M_{1}+1\left(M_{1}:\right.$ integer), and let $L_{2} \rightarrow \infty$. The centers of the unit cells are located at $x_{1}=-M_{1},-M_{1}+1, \ldots, M$, measured in the unit of $\left|a_{1}\right|$. For simplicity, we assume that the open system consists of an integer number of inversion-symmetric unit cells. Other cases are discussed in Sec. VI. As in the one-dimensional case, we replace the hopping amplitudes $t_{j}$ for all bonds that cross the boundary between $x_{1}=-M_{1}$ and $x_{1}=M_{1}$ by $\lambda t_{j}$, where $\lambda$ is real. Note that we impose a periodic boundary condition along the $x_{2}$ direction in the rest of this section. Figure 4 is a conceptual figure of a two-dimensional inversion-symmetric 
insulator cut at the boundary between $x_{1}=-M_{1}$ and $x_{1}=M_{1}$. For real $\lambda$, inversion symmetry is always preserved.

The inversion operator $\mathcal{P}$ changes the $2 \mathrm{D}$ wave vector $\boldsymbol{k}=\left(k_{1}, k_{2}\right)$ to $\left(-k_{1},-k_{2}\right)$. Let us focus on the 1D $\mathcal{P}$-invariant subspaces $k_{2}=0$ and $k_{2}=\pi$, which we call $\bar{\Gamma}$ and $\bar{Y}$, respectively. We can regard $\bar{\Gamma}$ and $\bar{Y}$ as 1D TRIM. Both of the subspaces can be considered as an effective 1D inversionsymmetric system. Let $N_{\bar{\Gamma}}$ and $N_{\bar{Y}}$ be the numbers of occupied states at $k_{2}=0$ and that of $k_{2}=\pi$, respectively. From Eq. (1), the change of $N_{\bar{\Gamma}}$ and $N_{\bar{Y}}(\bmod 2)$ by changing $\lambda=1$ to $\lambda=0$ is evaluated from $n_{-}\left(\Gamma_{j}\right)\left(\Gamma_{j} \in 2 \mathrm{D}\right.$ TRIM $)$ :

$$
\begin{aligned}
& \left.\left.N_{\bar{\Gamma}}\right|_{\lambda=0} \equiv N_{\bar{\Gamma}}\right|_{\lambda=1}+n_{-}(\Gamma)-n_{-}(X) \quad(\bmod 2), \\
& \left.\left.N_{\bar{Y}}\right|_{\lambda=0} \equiv N_{\bar{Y}}\right|_{\lambda=1}+n_{-}(Y)-n_{-}(M)(\bmod 2) .
\end{aligned}
$$

Since the system is insulating for $\lambda=1$, we get $\left.N_{\bar{\Gamma}}\right|_{\lambda=1}=$ $\left.N_{\bar{Y}}\right|_{\lambda=1}=L_{1} v$. By subtracting Eq. (13) from Eq. (12), we obtain the following equation:

$$
\begin{aligned}
\left.N_{\bar{\Gamma}}\right|_{\lambda=0}-\left.N_{\bar{Y}}\right|_{\lambda=0} & \equiv n_{-}(\Gamma)-n_{-}(X)-n_{-}(Y)+n_{-}(M) \\
& \equiv \sum_{\Gamma_{j} \in \text { TRIM }} n_{-}\left(\Gamma_{j}\right) \quad(\bmod 2) .
\end{aligned}
$$

From Eq. (14), when $\sum n_{-}\left(\Gamma_{j}\right)=1(\bmod 2)$, for the system with an open boundary condition, the parity of $N_{\bar{\Gamma}}$ is different from that of $N_{\bar{Y}}$. To compensate the difference, an odd number of chiral edge modes must exist between $\bar{\Gamma}$ and $\bar{Y}$.

Moreover as pointed out in Ref. [48], inversion parities at the respective TRIM have more information than their sum over all the TRIM. For example, if $\sum_{\Gamma_{j}} n_{-}\left(\Gamma_{j}\right)=1$, then we have two possibilities,

$$
\left(\left[N_{\bar{\Gamma}}\right]_{\lambda=1}^{\lambda=0},\left[N_{\bar{Y}}\right]_{\lambda=1}^{\lambda=0}\right) \equiv(1,0),(0,1) \quad(\bmod 2),
$$

and we can identify which possibility is realized from parity eigenvalues $n_{-}\left(\Gamma_{j}\right)$, from Eqs. (12) and (13). This indicates which of the 1D TRIM, $\bar{\Gamma}$ and $\bar{Y}$, is "inside of the boundary Fermi surface." Here, we define "inside of the boundary Fermi surface" as a subspace of $k$ space, where the number of occupied states is more or less than that of the bulk by an odd number. To express this feature, we define boundary fermion parity at $k_{y}$ to be $n_{\text {boundary }}\left(k_{y}\right)=\left[N_{k_{y}}\right]_{\lambda=1}^{\lambda=0}$, as an analog of the surface fermion parity in Ref. [48]. This boundary fermion parity $n_{\text {boundary }}\left(k_{y}\right)$ is always an integer. From Eqs. (12) and (13), we define the boundary fermion parity at $\overline{\Gamma_{j}}$ to be $\sum_{\Gamma_{i}} n_{-}\left(\Gamma_{i}\right)(\bmod 2)$ where the sum is taken over the $2 \mathrm{D}$ TRIM $\Gamma_{i}$ which is projected onto $\overline{\Gamma_{j}}(=\bar{\Gamma}, \bar{Y})$. As long as inversion symmetry is preserved, when we modify the system perturbatively without closing the gap, $n_{\text {boundary }}\left(\overline{\Gamma_{j}}\right)$ changes by an even number. Therefore, the parity of $n_{\text {boundary }}\left(\overline{\Gamma_{j}}\right)$ is a topological number, and it is natural that it is given by the parities at the 2D TRIM.

Here, we note the relation of our study and the previous works. Previous works [13-15] have shown that, for twodimensional systems, the quantity $(-1)^{\mathrm{Ch}}$, where $\mathrm{Ch}$ is the Chern number along a crystal plane of the system, is equal to the product of the inversion parities at TRIM on the plane in $\boldsymbol{k}$ space. Other studies [4,5] have shown that, for two-dimensional systems, the number of chiral edge modes is equal to the Chern number of the system. By combining these two results, we conclude that the quantity $(-1)^{N_{\text {edge }} \text {, }}$ where $N_{\text {edge }}$ is the number of chiral edge modes, is equal to the product of the inversion parities at TRIM. This is exactly the same statement which we have shown in this section. However, compared to the previous studies on bulkedge correspondence [4,5], our proof directly relates the bulk inversion parities to the existence of the chiral edge modes. Moreover, our proof clarifies that the inversion parities at each TRIM have more information than their product [13-15], leading to the notion of boundary fermion parity $n_{\text {boundary }}\left(\overline{\Gamma_{j}}\right)$. Therefore, our proof gives a new perspective to the existing results.

\section{B. Illustrative examples}

Here, we show two examples to see how the system evolves by the change of $\lambda$. Figure 5 shows two examples of the bulk parity and corresponding surface energy spectrum. Figure 5(b-1) shows the case with $\sum n_{-}\left(\Gamma_{j}\right)=0$. In this case, inversion parities at $\bar{\Gamma}$ and $\bar{Y}$ do not depend on $\lambda$, as shown in Figs. 5(b-2) and 5(b-3). Therefore, the corresponding surface energy spectrum, Fig. 5(b-4), does not have a chiral edge mode. Figure 5(c-1) shows the case with $\sum n_{-}\left(\Gamma_{j}\right)=1$. In this case, inversion parities at $\bar{\Gamma}$ depend on $\lambda$, as shown in Figs. 5(c-2) and 5(c-3). Therefore, when $\lambda=0$, the number of states at $\bar{\Gamma}$ is one less than that of bulk. However, inversion parities at $\bar{Y}$ do not depend on $\lambda$ as shown in Fig. 5(c2). Therefore, the corresponding surface energy spectrum, Fig. 5(c-4), have a chiral edge mode, which compensates the difference of the number of occupied states at $\bar{\Gamma}$ and $\bar{Y}$.

\section{BULK-HINGE CORRESPONDENCE IN 3D CLASS A}

In this section, we consider a three-dimensional noninteracting centrosymmetric insulator with periodic boundary conditions. In a three-dimensional system, there are eight TRIM. As in the case of one- and two-dimensional insulators, we have nine independent topological numbers $\left[v, n_{-}\left(\Gamma_{j}\right)\right]$, where $v$ is filling, and $n_{-}\left(\Gamma_{j}\right)$ is the number of occupied states with odd parity at the TRIM $\Gamma_{j}$. Interestingly, some combinations of $\left\{n_{-}\left(\Gamma_{j}\right)\right\}$ cannot be realized in atomic insulators. The following four numbers, calculated from $n_{-}\left(\Gamma_{j}\right)$, are indicators which specify whether the combinations of $\left\{n_{-}\left(\Gamma_{j}\right)\right\}$ can be realized in an atomic insulator or not [50-52]:

$$
\begin{aligned}
\mu_{1} & =\sum_{\Gamma_{j}: \text { TRIM }} \frac{n_{+}\left(\Gamma_{j}\right)-n_{-}\left(\Gamma_{j}\right)}{2} \\
& \equiv-\sum_{\Gamma_{j}: \text { TRIM }} n_{-}\left(\Gamma_{j}\right) \quad(\bmod 4), \\
v_{a} & \equiv \sum_{\Gamma_{j}: \text { TRIM } \wedge k_{a}=\pi} n_{-}\left(\Gamma_{j}\right) \quad(\bmod 2)(a=1,2,3) .
\end{aligned}
$$

We note that, for insulators, $\mu_{1}$ takes only the values 0 or 2 . If $\mu_{1}=1$ or 3 , then there are Weyl points somewhere in $\boldsymbol{k}$ space between the valence bands and the conduction bands, meaning that the system is not an insulator $[13,14]$.

In this section, we show a direct correspondence between the bulk topological invariant $\mu_{1}$ and the existence of chiral 
(a)

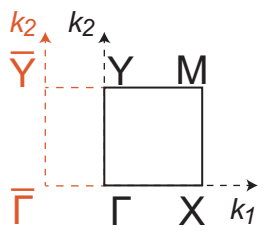

$(b-1)$

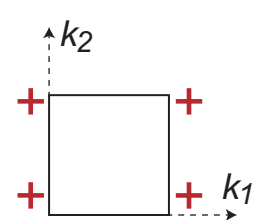

$(\mathrm{b}-2)$

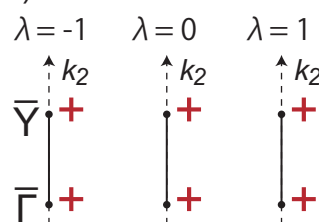

$(\mathrm{b}-3)$

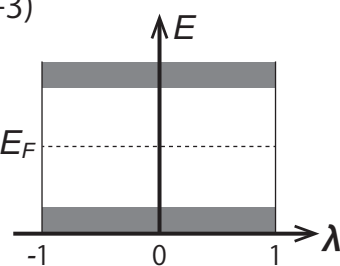

$(b-4)$
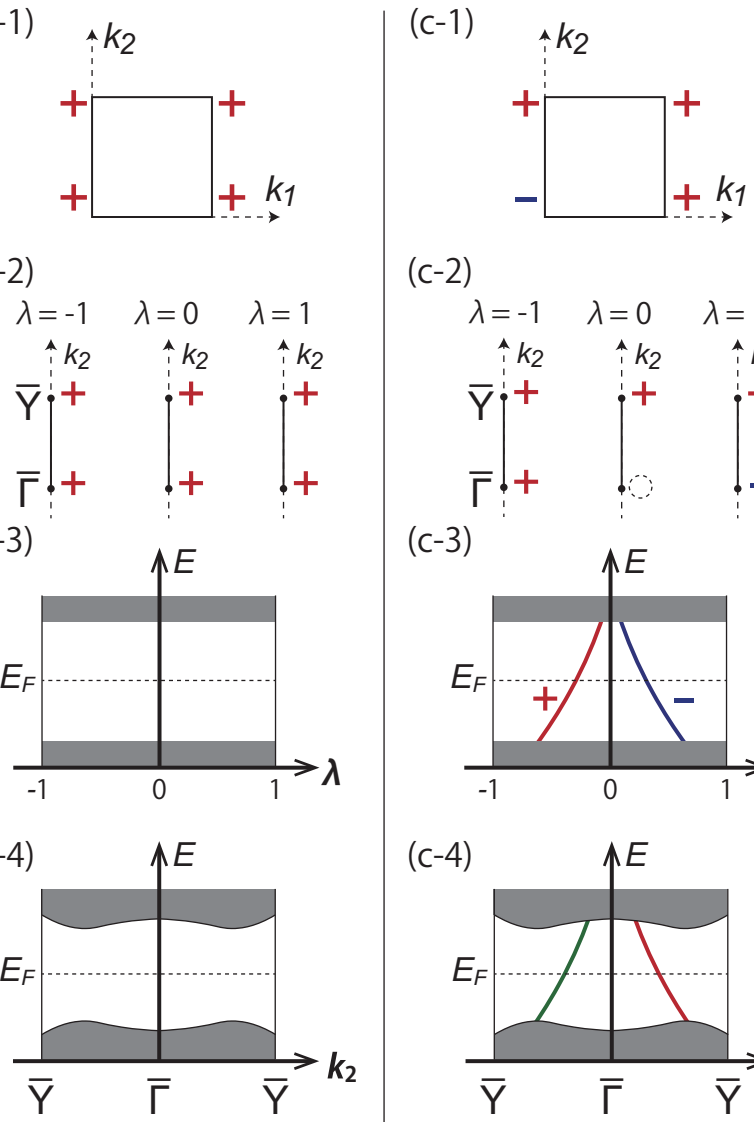

(c-2)

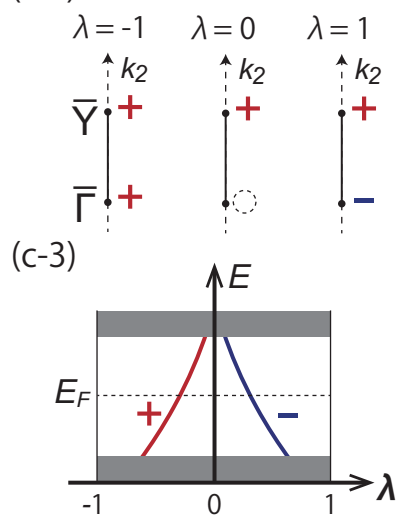

(c-4)

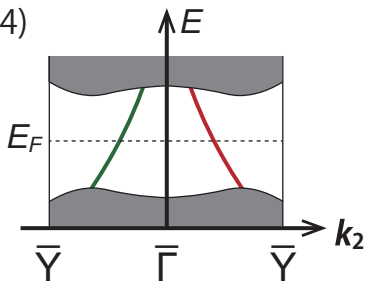

FIG. 5. Two representative examples of bulk parities and corresponding energy spectra. (a) Quarter of the 2D Brillouin zone with the symmetry labels shown in black, and a half of the projected 1D Brillouin zone with the symmetry labels shown in orange. (b), (c) Two examples of inversion parities and resulting spectra. (b-1)-(b4) and (c-1)-(c-4) show two different cases. (b-1), (c-1) Inversion parities at 2D TRIM. (b-2), (c-2) Parity arrangements at $\bar{\Gamma}$ and $\bar{Y}$ for $\lambda=-1,0,1$. In addition to the state whose parity is shown in (b-2) and (c-2), there are $\frac{\left(L_{1}-1\right) v}{2}$ states with even parity and $\frac{\left(L_{1}-1\right) v}{2}$ states with odd parity, and they are not shown in the figure for simplicity. In (c-2), at $\lambda=0$, the parity at $\bar{\Gamma}$ is blank, because the number of occupied states is one less than that of the bulk. (b-3), (c-3) Energy spectra at $\bar{\Gamma}$ in the process of changing $\lambda$. (b-4), (c-4) Energy spectrum. In $(\mathrm{c}-4), N_{\bar{\Gamma}} \neq N_{\bar{Y}}(\bmod 2)$, and an odd number of chiral edge modes exist.

hinge states. To this end, we introduce a cutting procedure along one direction, which we call $x_{1}$ axis, and study the $2 \mathrm{D}$ system after cutting the system along a plane $x_{1}=$ const., which corresponds to $\lambda=0$ in the cutting process. We show that in a system with an open boundary condition in one direction, when $\mu_{1}=2$, (i) the surface band structure is gapless or (ii) the surface band structure is gapped and the Chern number of the system is equal to $1(\bmod 2)$. More precisely, under the

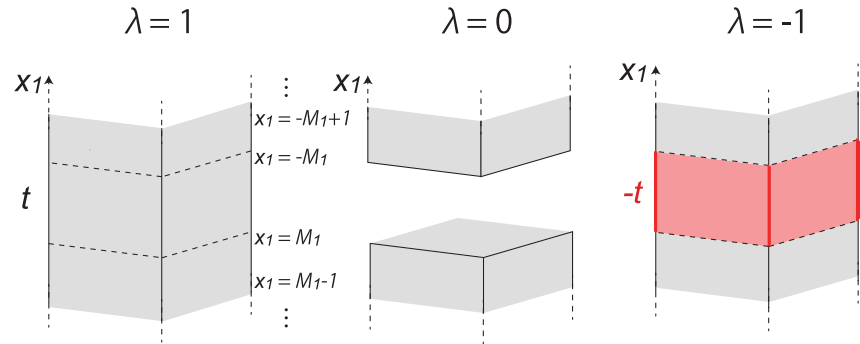

FIG. 6. Conceptual figure of a three-dimensional inversionsymmetric insulator cut at the boundary between $x_{1}=M_{1}$ and $x_{1}=$ $-M_{1}$. When $\lambda=1$, the system is periodic, and when $\lambda=0$ the system is an open finite system along the $x_{1}$ direction. When $\lambda=-1$, this system is antiperiodic along the $x_{1}$ direction.

assumption that the system is insulating both in the bulk and the surface, we show the following relation:

$$
\left.\sum_{\overline{\Gamma_{j}}} \tilde{n}_{-}\left(\overline{\Gamma_{j}}\right)\right|_{\lambda=0} \equiv \frac{1}{2}\left(-\mu_{1}\right) \quad(\bmod 2),
$$

where $\tilde{n}_{-}\left(\overline{\Gamma_{j}}\right)$ is the number of occupied states with odd parity at the 2D TRIM $\overline{\Gamma_{j}}$. Equation (18) is a central result of this paper, which shows the direct relationship of the 3D indicator in the bulk and the 2D indicator with an open boundary.

\section{A. General proof}

We set the system size along the $x_{i}$ axis to be $L_{i}(i=$ $1,2,3)$ measured in units of $\left|\boldsymbol{a}_{i}\right|(i=1,2,3)$. For simplicity, let $L_{1}$ be an odd integer, $L_{1}=2 M_{1}+1\left(M_{1}:\right.$ integer), and let $L_{2}, L_{3} \rightarrow \infty$. The centers of the unit cells are located at $x_{1}=-M_{1},-M_{1}+1, \ldots, M_{1}$, measured in the units of $\left|\boldsymbol{a}_{1}\right|$. We first start with a periodic system with $x_{1}=M_{1}$ and $x_{1}=-M_{1}$ being connected. We then replace the hopping amplitudes $t_{j}$ for all bonds that cross the boundary between $x_{1}=M_{1}$ and $x_{1}=-M_{1}$ by $\lambda t_{j}$, where $\lambda$ is real. Note that we impose a periodic boundary condition for $x_{2}$ and $x_{3}$ directions in the rest of this section. Figure 6 is a conceptual figure of a three-dimensional inversion-symmetric insulator cut at the boundary between $x_{1}=M_{1}$ and $x_{1}=-M_{1}$. For any real values of $\lambda$, inversion symmetry is always preserved. For simplicity, we assume that the open system consists of an integer number of inversion-symmetric unit cells. Other cases are discussed in Sec. VI.

By considering the degree of freedom in the $x_{1}$ direction as an internal degree of freedom, this system can be regarded as a two-dimensional system. Then we regard $\boldsymbol{k}_{\|} \equiv\left(k_{2}, k_{3}\right)^{T}$ as a wave vector in this effective 2D system, where $k_{2}$ and $k_{3}$ take the value $-\pi<k_{i} \leqslant \pi(i=2,3) . \boldsymbol{k}_{\|}$is a TRIM if and only if $\boldsymbol{k}_{\|}=(0,0),(\pi, 0),(0, \pi),(\pi, \pi)$, which we call $\bar{\Gamma}, \bar{Y}$, $\bar{Z}, \bar{T}$, respectively. Let $N_{\overline{\Gamma_{j}}}(\lambda)$ be the total number of occupied states at $\boldsymbol{k}_{\|}=\overline{\Gamma_{j}}$ for a given value of $\lambda$.

We show that $\left.\tilde{n}_{-}\left(\overline{\Gamma_{j}}\right)\right|_{\lambda=0}$ is evaluated from the knowledge of $n_{-}\left(\Gamma_{j}\right)$ and $\left.N_{\overline{\Gamma_{j}}}\right|_{\lambda=0}$. We note that $\boldsymbol{k}_{\|}=\overline{\Gamma_{j}}$ represents a one-dimensional $\mathcal{P}$-invariant subspace in 3D $\boldsymbol{k}$ space. In this subspace, $\tilde{n}_{-}\left(\overline{\Gamma_{j}}\right)$ corresponds to $N_{-}$in the one-dimensional 

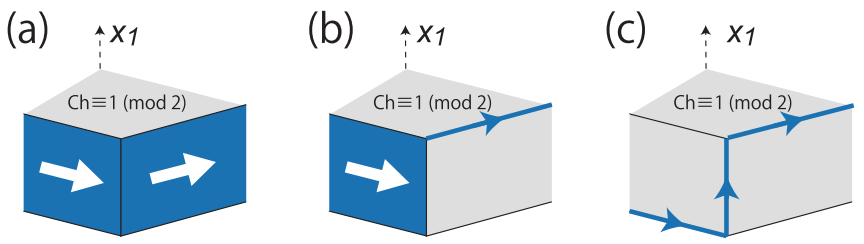

FIG. 7. Conceptual figure of positions of gapless surface states and hinge states for $\mu_{1}=2$. In this case, when the system is cut along the $x_{2}-x_{3}$ plane, the resulting $2 \mathrm{D}$ system has the Chern number equal to $1(\bmod 2)$, meaning that the $2 \mathrm{D}$ system has gapless chiral edge states. These chiral edge states as a 2D system can be either surface states or hinge states. In (a), the gapless chiral states are surface states at the side surfaces. In (b), the gapless chiral states are composed of surfaces states and hinge states. In (c), they are hinge states.

case discussed in Sec. II. Therefore, from Eqs. (3), (5), and (10), the following equations hold:

$$
\begin{gathered}
\left.\tilde{n}_{-}\left(\overline{\Gamma_{j}}\right)\right|_{\lambda=1}=\frac{\left(L_{1}-1\right) v}{2}+n_{-}\left(0, \overline{\Gamma_{j}}\right), \\
\left.\tilde{n}_{-}\left(\overline{\Gamma_{j}}\right)\right|_{\lambda=-1}=\frac{\left(L_{1}-1\right) v}{2}+n_{-}\left(\pi, \overline{\Gamma_{j}}\right), \\
{\left[\tilde{n}_{-}\left(\overline{\Gamma_{j}}\right)\right]_{\lambda=1}^{\lambda=0}=\frac{1}{2}\left[N_{\overline{\Gamma_{j}}}\right]_{\lambda=1}^{\lambda=0}+\frac{n_{-}\left(\pi, \overline{\Gamma_{j}}\right)-n_{-}\left(0, \overline{\Gamma_{j}}\right)}{2},}
\end{gathered}
$$

where $\left(0, \overline{\Gamma_{j}}\right)$ and $\left(\pi, \overline{\Gamma_{j}}\right)$ are 3D TRIM. By adding Eq. (19) and Eq. (21), we get the following equation:

$$
\left.\tilde{n}_{-}\left(\overline{\Gamma_{j}}\right)\right|_{\lambda=0}=\frac{N_{\overline{\Gamma_{j}}} \mid \lambda=0-v}{2}+\frac{n_{-}\left(0, \overline{\Gamma_{j}}\right)+n_{-}\left(\pi, \overline{\Gamma_{j}}\right)}{2},
$$

where we use the relation $\left.N_{\overline{\Gamma_{j}}}\right|_{\lambda=1}=L_{1} v$. By summing Eq. (22) over $\overline{\Gamma_{j}}$, we obtain the following equation:

$$
\left.\sum_{\overline{\Gamma_{j}}} \tilde{n}_{-}\left(\overline{\Gamma_{j}}\right)\right|_{\lambda=0}=\left.\frac{1}{2} \sum_{\overline{\Gamma_{j}}} N_{\overline{\Gamma_{j}}}\right|_{\lambda=0}-2 v+\frac{1}{2} \sum_{\Gamma_{j}} n_{-}\left(\Gamma_{j}\right) .
$$

Next, let us assume that the system at $\lambda=0$ is also insulating, which means that the surface is also insulating. Then the values of $\left.N_{\overline{\Gamma_{j}}}\right|_{\lambda=0}$ at the four TRIM are equal, i.e., $\left.N_{\overline{\Gamma_{j}}}\right|_{\lambda=0}=$ $N_{\mathrm{OBC}}$. Then, we get the following equation:

$$
\left.\sum_{\overline{\Gamma_{j}}} \tilde{n}_{-}\left(\overline{\Gamma_{j}}\right)\right|_{\lambda=0}=2 N_{\mathrm{OBC}}-2 v+\frac{1}{2}\left(-\mu_{1}\right) .
$$

Then we note that the left-hand side of Eq. (24) is equal to the parity of the Chern number at $\lambda=0$. It is because, in a two-dimensional centrosymmetric system, $(-1)^{\mathrm{Ch}}$ is equal to the product of the $\mathcal{P}_{2 \mathrm{D}}$ eigenvalues at TRIM, where $\mathcal{P}_{2 \mathrm{D}}$ is the inversion operator of the two-dimensional system, as shown in the Sec. III and in some previous works [13-15]. Equation (18) is derived by taking modulo 2 in Eq. (24).

In particular, when $\mu_{1}=2$, we conclude that (Chern) $\left.\right|_{\lambda=0}=1(\bmod 2)$. It means that if we make the system to be open also along $x_{2}$ and $x_{3}$ directions, this system supports chiral edge modes with the number of chiral modes being an odd number. These chiral modes can be on surfaces or on hinges, as shown in Figs. 7(a)-7(c). If we further assume that all the surfaces are gapped, then these chiral modes are localized on the hinges.

\section{B. Illustrative examples}

In this subsection, we consider two typical examples and demonstrate the validity of Eq. (18) holds: (A) $\mu_{1}=2, v_{a}=0$ (HOTI) and (B) $\mu_{1}=2, v_{1}=1, v_{2}=v_{3}=0$ (weak Chern insulator). In the rest of this subsection, we assume that the surface band structure, i.e., the band structure at $\lambda=0$, is gapped. Since $\mu_{1}=2$ in both cases, from Eq. (18), both systems have a nontrivial Chern number when $\lambda=0$. However, due to the difference of the weak topological number, $v_{1}$, their topological nature is completely different. Here, we illustrate the spectral flow for the change of $\lambda$, and we confirm the result by a model calculation. We find that the spectral flows of (A) and (B) are completely different. The difference comes from the difference of $v_{1}$.

$$
\text { 1. } \mu_{1}=2, v_{a}=0(a=1,2,3)
$$

One of the simplest examples to realize $\mu_{1}=2, v_{a}=$ $0(a=1,2,3)$ is $n_{-}(\Gamma)=2, n_{-}\left(\Gamma_{j}\right)=0\left(\Gamma_{j} \neq \Gamma\right)$ and $v=$ 2 . The bulk parity corresponding to this example is illustrated in Fig. 8(b). We note that, since $v=2$ and $L_{1}-1=2 M_{1}$, from Eqs. (19) and (20), $\left.\tilde{n}_{-}\left(\overline{\Gamma_{j}}\right)\right|_{\lambda=1}=2 M_{1}+n_{-}\left(0, \overline{\Gamma_{j}}\right)$, and $\left.\tilde{n}_{-}\left(\overline{\Gamma_{j}}\right)\right|_{\lambda=-1}=2 M_{1}+n_{-}\left(\pi, \overline{\Gamma_{j}}\right)$. Then the following relations hold:

$$
\begin{gathered}
\left.\tilde{n}_{-}(\bar{\Gamma})\right|_{\lambda=1}=2 M_{1}+2,\left.\quad \tilde{n}_{-}(\bar{\Gamma})\right|_{\lambda=-1}=2 M_{1}, \\
\left.\tilde{n}_{-}(\bar{Y})\right|_{\lambda= \pm 1}=\left.\tilde{n}_{-}(\bar{Z})\right|_{\lambda= \pm 1}=\left.\tilde{n}_{-}(\bar{T})\right|_{\lambda= \pm 1}=2 M_{1} .
\end{gathered}
$$

Next we calculate $\left.\tilde{n}_{-}\left(\overline{\Gamma_{j}}\right)\right|_{\lambda=0}$ for TRIM $\overline{\Gamma_{j}}$. First, let us consider the case of $\boldsymbol{k}_{\|}=\bar{\Gamma}$. From Eq. (25), when $\lambda$ is decreased from 1 to -1 , two odd-parity states cross the Fermi energy $E_{F}$ from below, and two even parity states cross $E_{F}$ from above. Furthermore, as we show in, the energy spectrum is symmetric under the flipping of the sign of $\lambda$, and the bound states $\left|\psi_{l}(\lambda)\right\rangle$ and $\left|\psi_{l}(-\lambda)\right\rangle$ have opposite parities. Figures $8(\mathrm{~d})$ and $8(\mathrm{e})$ shows two representative examples of the energy spectrum which satisfy these requirements. The number of states below the Fermi energy $E_{F}$ at $\lambda=0,\left.N_{\bar{\Gamma}}\right|_{\lambda=0}$, is equal to that of bulk in Fig. 8(d) and two less than that of bulk in Fig. 8(e). The differences in the value of $\tilde{n}_{-}(\bar{\Gamma})$ between $\lambda=0$ and $\lambda=1$ are $\left[\tilde{n}_{-}(\bar{\Gamma})\right]_{\lambda=1}^{\lambda=0}=-1$ in Fig. $8(\mathrm{~d})$ and $\left[\tilde{n}_{-}(\bar{\Gamma})\right]_{\lambda=1}^{\lambda=0}=-2$ in Fig. 8(e). Therefore, $\left.\tilde{n}_{-}(\bar{\Gamma})\right|_{\lambda=0} \equiv 1$ $(\bmod 2)$ in Fig. $8(\mathrm{~d})$, and $\left.\tilde{n}_{-}(\bar{\Gamma})\right|_{\lambda=0} \equiv 0(\bmod 2)$ in Fig. 8(e).

Next, let us consider the case of $\boldsymbol{k}_{\|}=\bar{Y}$. We note that the cases of $\boldsymbol{k}_{\|}=\bar{Z}, \bar{T}$ are the same as the case of $\boldsymbol{k}_{\|}=\bar{Y}$. We consider the energy spectrum at $\bar{Y}$ in changing $\lambda$ from 1 to -1 . Since $\left.\tilde{n}_{-}(\bar{Y})\right|_{\lambda= \pm 1}=2 M_{1}$, the number of states with even (odd) parity which cross $E_{F}$ from above is equal to that from below through the change of $\lambda$ from $\lambda=1$ to $\lambda=-1$. Figures $8(\mathrm{f})$ and $8(\mathrm{~g})$ show two representative examples of energy spectrum which satisfy this requirement. In Fig. 8(f), $\left.N_{\bar{Y}}\right|_{\lambda=0}$ is equal to that of bulk, and in Fig. $8(\mathrm{~g}),\left.N_{\bar{Y}}\right|_{\lambda=0}$ is two less than that of bulk. The differences in the value of $\tilde{n}_{-}(\bar{Y})$ between $\lambda=0$ and $\lambda=1$ are $\left[\tilde{n}_{-}(\bar{Y})\right]_{\lambda=1}^{\lambda=0}=0$ in Fig. 8(f) and $\left[\tilde{n}_{-}(\bar{Y})\right]_{\lambda=1}^{\lambda=0}=-1$ in Fig. $8(\mathrm{~g})$. Therefore, $\left.\tilde{n}_{-}(\bar{Y})\right|_{\lambda=0} \equiv 0$ $(\bmod 2)$ in Fig. 8(f), and $\left.\tilde{n}_{-}(\bar{Y})\right|_{\lambda=0} \equiv 1(\bmod 2)$ in Fig. $8(\mathrm{~g})$. 
(a)

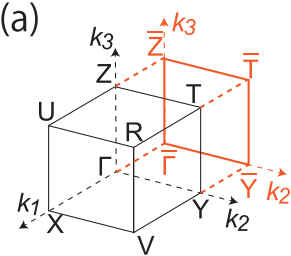

(d)

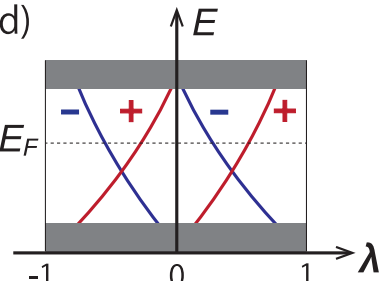

(f)

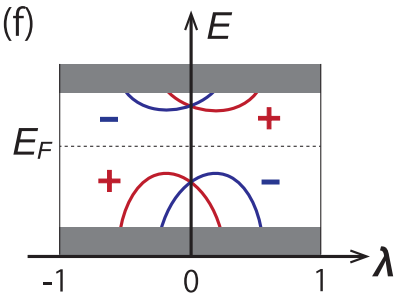

(h) $\lambda=-1$

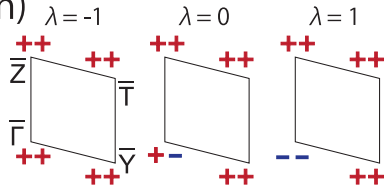

(b) $k_{3}$

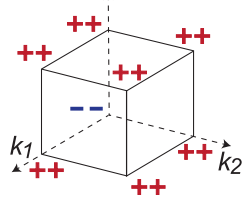

(e)

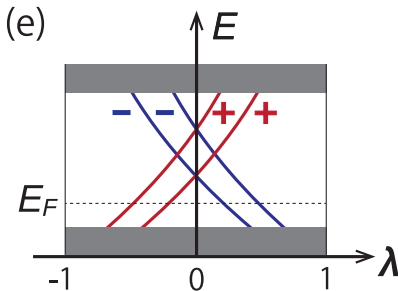

(g)

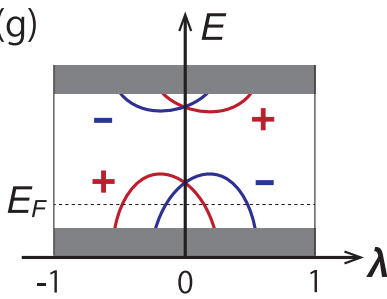

(i) $\lambda=-1 \quad \lambda=0 \quad \lambda=1$

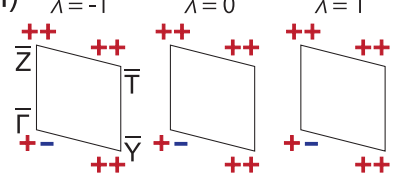

FIG. 8. Illustrative examples of $\mu_{1}=2$ topological insulators. (a) $1 / 8$ of the $3 \mathrm{D}$ Brillouin zone and the symmetry labels shown in black, and $1 / 4$ of the 2D Brillouin zone and the symmetry labels shown in orange. (b), (c) Two examples of inversion parities at TRIM for (b) $v_{a}=0$ (HOTI) and (c) $v_{1}=1, v_{2}=v_{3}=0$ (weak Chern insulator). (d)-(g) Four representative examples of the energy spectrum on $\lambda$. Red and blue lines represent bound states with even and odd parity, respectively. (f) and (g) differ only by the Fermi energy. (h), (i) Two examples of inversion parities at 2D TRIM corresponding to (b) and (c), respectively. In every case, in addition to the state whose parity is shown in (h) and (i), there are $\frac{\left(L_{1}-1\right) v}{2}$ states with even parity and $\frac{\left(L_{1}-1\right) v}{2}$ states with odd parity, and they are not shown in the figure for simplicity. When $\lambda=0$, both examples have one state with odd parity at $\bar{\Gamma}$.

Finally, we combine the results at $\bar{\Gamma}, \bar{Y}, \bar{Z}$, and $\bar{T}$. First of all, from the assumption that the bulk is insulating, the number of bulk occupied states is equal for all the 2D TRIM $\overline{\Gamma_{j}}$ :

$$
\left.\left.N\right|_{\lambda=1} \equiv N_{\bar{\Gamma}}\right|_{\lambda=1}=\left.N_{\bar{Y}}\right|_{\lambda=1}=\left.N_{\bar{Z}}\right|_{\lambda=1}=\left.N_{\bar{T}}\right|_{\lambda=1} .
$$

Second, from the assumption that the surface is insulating, the following equation holds:

$$
\left.\left.N\right|_{\lambda=0} \equiv N_{\bar{\Gamma}}\right|_{\lambda=0}=\left.N_{\bar{Y}}\right|_{\lambda=0}=\left.N_{\bar{Z}}\right|_{\lambda=0}=\left.N_{\bar{T}}\right|_{\lambda=0} .
$$

Hence, there are two possibilities, depending on the difference between $\left.N\right|_{\lambda=1}$ and $\left.N\right|_{\lambda=0}$ being 0 or 2 modulo 4. This constrains combinations of evolutions of states at $\bar{\Gamma}, \bar{Y}, \bar{Z}$ and $\bar{T}$ upon a change of $\lambda$. First, when $\left.N\right|_{\lambda=1}=$ $\left.N\right|_{\lambda=0}$, the spectrum at $\bar{\Gamma}$ is like Fig. $8(\mathrm{~d})$, while that at $\bar{Y}, \bar{Z}$ and $\bar{T}$ are Fig. 8(f). Therefore, $\left.\tilde{n}_{-}(\bar{\Gamma})\right|_{\lambda=0} \equiv 1$ and $\left.\left.\left.\tilde{n}_{-}(\bar{Y})\right|_{\lambda=0} \equiv \tilde{n}_{-}(\bar{Z})\right|_{\lambda=0} \equiv \tilde{n}_{-}(\bar{T})\right|_{\lambda=0} \equiv 0(\bmod 2)$, and the
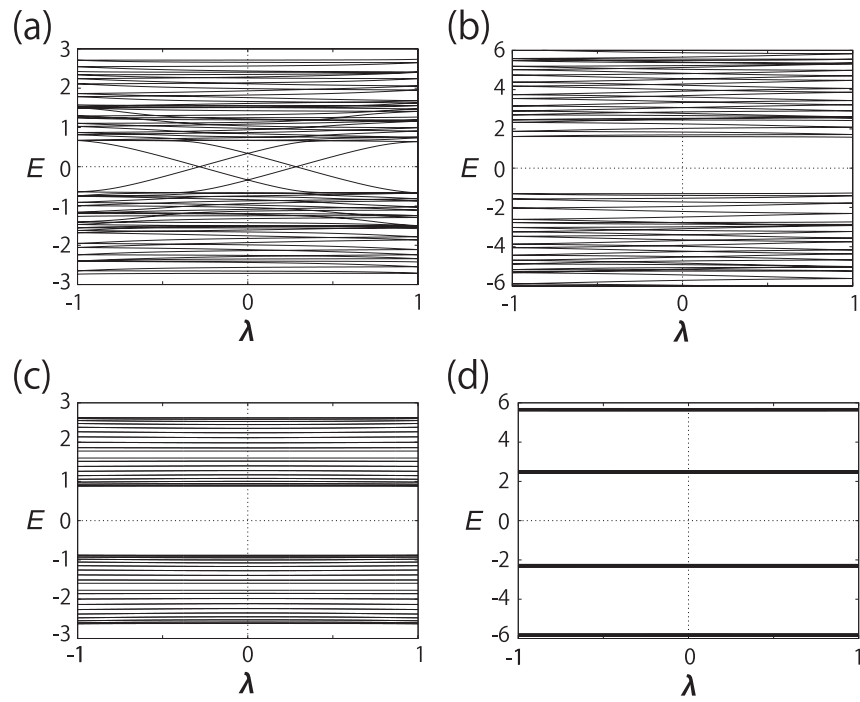

FIG. 9. Model calculations for two examples of $\mu_{1}=2$ topological insulators, (a), (b) a HOTI and (c), (d) a weak Chern insulator corresponding to cases (A) and (B) in Sec. IV B. We set $L_{1}=19$. (a), (b) Energy spectrum of the model Eq. (29) for a HOTI as a function of $\lambda$ at (a) $\bar{\Gamma}$ and (b) $\bar{Y}$. (c), (d) Energy spectrum of the model Eq. (30) for a weak Chern insulator as a function of $\lambda$ at (c) $\bar{\Gamma}$ and (d) $\bar{Y}$.

sum of the number of states with odd parity at 2D TRIM $\overline{\Gamma_{j}}$ is $1(\bmod 2)$. Figure $8(\mathrm{~h})$ shows inversion parities at $\lambda=-1,0$, and 1, corresponding to Figs. 8(d) and 8(f). Second, when $\left.N\right|_{\lambda=1}-\left.N\right|_{\lambda=0} \equiv 2(\bmod 4)$, the spectrum at $\bar{\Gamma}$ is Fig. 8(e), while that at $\bar{Y}, \bar{Z}$ and $\bar{T}$ are Fig. 8(g). Therefore, $\left.\tilde{n}_{-}(\bar{\Gamma})\right|_{\lambda=0} \equiv 0$ and $\left.\left.\left.\tilde{n}_{-}(\bar{Y})\right|_{\lambda=0} \equiv \tilde{n}_{-}(\bar{Z})\right|_{\lambda=0} \equiv \tilde{n}_{-}(\bar{T})\right|_{\lambda=0} \equiv$ 1 , and their sum is $3 \equiv 1(\bmod 2)$. To summarize, in either case, $\left.\sum_{\overline{\Gamma_{j}}} \tilde{n}_{-}\left(\overline{\Gamma_{j}}\right)\right|_{\lambda=0}=1(\bmod 2)$, which means that the Chern number of the system is $1(\bmod 2)$.

We confirm this by a model calculation. We use the following tight-binding Hamiltonian:

$$
\begin{aligned}
\mathcal{H}(\boldsymbol{k})= & -\left(m-c \sum_{j=x, y, z} \cos k_{j}\right) \tau_{z} \\
& -t \sum_{j=x, y, z} \tau_{x} \sigma_{j} \sin k_{j}+\boldsymbol{B} \cdot \sigma+A \tau_{z} \sigma_{z},
\end{aligned}
$$

where $m=4, c=2, t=1, \boldsymbol{B}=(0.3,0.3,0.3)$ and $A=0.3$. This Hamiltonian is symmetric under inversion operation $\mathcal{P}=$ $\tau_{z}$, and the inversion parities at TRIM are shown in Fig. 8(b). If $\boldsymbol{B}=\mathbf{0}$ and $A=0$, then the model Eq. (29) describes a conventional topological insulator protected by time-reversal symmetry $[40,53]$. $\boldsymbol{B}$ is considered as a uniform magnetic field, which breaks time-reversal symmetry. $A$ is considered as an orbital-dependent magnetic field which depends on the orbital degrees of freedom $\tau$. The Fermi energy is set to be $E_{F}=0$. The energy spectrum on $\lambda$ at $\bar{\Gamma}$ and $\bar{Y}$ is shown in Figs. 9(a) and 9(b), respectively. As expected from the theoretical calculation, the energy spectrum at $\bar{\Gamma}$ has crossing points as shown in Fig. 9(a), and there is no crossing point at $\bar{Y}$ as shown in Fig. 9(b). This corresponds to the case with Figs. 8(d) and 8(f). These results support our theoretical calculation. 


$$
\text { 2. } \mu_{1}=2, v_{1}=1, v_{2}=v_{3}=0
$$

As an example to realize $\mu_{1}=2, v_{1}=1, v_{2}=v_{3}=0$, here we take $n_{-}(\Gamma)=n_{-}(X)=1, n_{-}\left(\Gamma_{j}\right)=0\left(\Gamma_{j} \neq \Gamma, X\right)$, and $v=2$. The bulk parity corresponding to this example is illustrated in Fig. 8(c).

First, we note that the cases of $\boldsymbol{k}_{\|}=\bar{Y}, \bar{Z}$, and $\bar{T}$ are the same as the case of $\boldsymbol{k}_{\|}=\bar{Y}$ in the previous example in Sec. IV B1. Therefore, for $\overline{\Gamma_{j}}=\bar{Y}, \bar{Z}$, and $\bar{T}$, we get $\left.\tilde{n}_{-}\left(\overline{\Gamma_{j}}\right)\right|_{\lambda=0} \equiv 0(\bmod 2)$ in Fig. $8(\mathrm{f})$, and $\left.\tilde{n}_{-}\left(\overline{\Gamma_{j}}\right)\right|_{\lambda=0} \equiv 1$ $(\bmod 2)$ in Fig. $8(\mathrm{~g})$.

Next, let us consider the case of $\boldsymbol{k}_{\|}=\bar{\Gamma}$. Since $v=$ 2 and $L_{1}-1=2 M_{1}$, from Eqs. (19) and (20), we get $\left.\tilde{n}_{-}(\bar{\Gamma})\right|_{\lambda=1}=2 M_{1}+n_{-}(\Gamma)=2 M_{1}+1$, and $\left.\tilde{n}_{-}(\bar{\Gamma})\right|_{\lambda=-1}=$ $2 M_{1}+n_{-}(X)=2 M_{1}+1$. Therefore, through a change from $\lambda=1$ to $\lambda=-1$, the number of states with even (odd) parity which cross $E_{F}$ from above is equal to that from below as a whole. Two examples of the possible energy spectra are shown in Figs. $8(\mathrm{f})$ and $8(\mathrm{~g})$. We get $\left.\tilde{n}_{-}(\bar{\Gamma})\right|_{\lambda=0} \equiv 1(\bmod 2)$ in Fig. 8(f), and $\left.\tilde{n}_{-}(\bar{\Gamma})\right|_{\lambda=0} \equiv 0(\bmod 2)$ in Fig. $8(\mathrm{~g})$.

Finally, we combine the results at $\bar{\Gamma}, \bar{Y}, \bar{Z}$, and $\bar{T}$. First of all, from the assumption that both the bulk and the surface is insulating, $\left.N_{\overline{\Gamma_{j}}}\right|_{\lambda=0}$ and $\left.N_{\overline{\Gamma_{j}}}\right|_{\lambda=1}$ do not depend on $\overline{\Gamma_{j}}$. Therefore, in Fig. 8, within the two cases, Figs. 8(f) and 8(g), the energy spectra at the four $2 \mathrm{D}$ TRIM $\overline{\Gamma_{j}}\left(\overline{\Gamma_{j}}=\bar{\Gamma}, \bar{Y}, \bar{Z}, \bar{T}\right)$ are the same, because $N_{\lambda=1}-N_{\lambda=0}$ is equal to $0(\bmod 4)$ in Fig. 8(f) and $2(\bmod 4)$ in Fig. $8(\mathrm{~g})$. In Fig. $8(\mathrm{f})$, since $\left.\tilde{n}_{-}(\bar{\Gamma})\right|_{\lambda=0} \equiv 1$ and $\left.\left.\left.\tilde{n}_{-}(\bar{Y})\right|_{\lambda=0} \equiv \tilde{n}_{-}(\bar{Z})\right|_{\lambda=0} \equiv \tilde{n}_{-}(\bar{T})\right|_{\lambda=0} \equiv 0$, their sum is 1 $(\bmod 2)$. Figure $8(i)$ shows inversion parities at $\lambda=-1,0$ and 1, corresponding to Fig. 8(f). In Fig. $8(\mathrm{~g})$, since $\left.\tilde{n}_{-}(\bar{\Gamma})\right|_{\lambda=0} \equiv$ 0 and $\left.\left.\left.\tilde{n}_{-}(\bar{Y})\right|_{\lambda=0} \equiv \tilde{n}_{-}(\bar{Z})\right|_{\lambda=0} \equiv \tilde{n}_{-}(\bar{T})\right|_{\lambda=0} \equiv 1$, their sum is $3 \equiv 1(\bmod 2)$. In either case, $\left.\sum_{\overline{\Gamma_{j}}} \tilde{n}_{-}\left(\overline{\Gamma_{j}}\right)\right|_{\lambda=0}=1(\bmod$ 2 ), which means that the Chern number of the system is 1 $(\bmod 2)$.

We confirm this by model calculation. We use the following tight-binding Hamiltonian:

$$
\begin{aligned}
\mathcal{H}(\boldsymbol{k})= & -\left(m-c \sum_{j=y, z} \cos k_{j}\right) \tau_{z} \\
& -t \sum_{j=x, y, z} \tau_{x} \sigma_{j} \sin k_{j}+\boldsymbol{B} \cdot \sigma+A \tau_{z} \sigma_{z},
\end{aligned}
$$

where $m=4, c=2, t=1, \boldsymbol{B}=(1.5,0.5,0.5)$, and $A=0.3$. This Hamiltonian is symmetric under inversion operation $\mathcal{P}=\tau_{z}$, and the inversion parities at TRIM are shown in Fig. 8(c). The main difference between the model Eqs. (29) and (30) is presence/absence of the term $\tau_{z} \cos k_{x}$. The energy spectrum on $\lambda$ at $\bar{\Gamma}$ and $\bar{Y}$ is shown in Figs. 9(c) and 9(d), respectively. As expected from the theoretical calculation, the energy spectrum at $\bar{\Gamma}$ and $\bar{Y}$ have no crossing point as shown in Figs. 9(c) and 9(d), respectively. From these calculations, in the case with $\left(\mu_{1}, v_{1}\right)=(2,1)$, we conclude that the Chern number perpendicular to the $x_{1}$ axis is always 1: $\left.($ Chern $)\right|_{\lambda=1}=\left.($ Chern $)\right|_{\lambda=0}=1(\bmod 2)$. This is different from the previous example with $\left(\mu_{1}, v_{1}\right)=(2,0)$. In that case, the Chern number depends on $\lambda:\left.($ Chern $)\right|_{\lambda=1}=0(\bmod 2)$ and $\left.($ Chern $)\right|_{\lambda=0}=1(\bmod 2)$.

\section{Comparison to the layer-reduction approach}

In this subsection, we discuss the layer-reduction approach in Ref. [40], which says that HOTIs can be smoothly connected to conventional TIs in a lower dimension. While this conclusion in Ref. [40] looks similar to ours, this approach is not directly applied to general systems. Below we give a model of a 3D HOTI, which is difficult to handle with the layer-reduction approach [40]. In Ref. [40], the authors showed that a 3D HOTI slab is adiabatically connected to a 2D Chern insulator, by using a model and reducing the thickness of the HOTI slab. Then they found that the resulting one-layer insulator is a Chern insulator. However, as we show below, some HOTIs do not have this property.

Here, we show a model of a 3D HOTI, whose single-layer insulator is a trivial insulator. We consider the following tightbinding Hamiltonian:

$$
\begin{aligned}
\mathcal{H}(\boldsymbol{k})= & -t \sum_{j=x, y, z} \tau_{x} \sigma_{j} \sin k_{j} \\
& -\cos k_{z}\left(m-c \sum_{j=x, y, z} \cos k_{j}\right) \tau_{z}+\boldsymbol{B} \cdot \boldsymbol{\sigma},
\end{aligned}
$$

where $t=c=1, \quad m=2$, and $\boldsymbol{B}=(0,0.25,0.25)$. This Hamiltonian is invariant by the inversion operation $\mathcal{P}=\tau_{z}$, and the bulk inversion parities of the two valence bands at TRIM are shown in Fig. 10(a). Since the $Z_{4}$ indicator is $\mu_{1}=2$, this Hamiltonian describes a HOTI. One can rewrite this Hamiltonian in a matrix form as

$$
H=\left(\begin{array}{cccccc}
\ddots & \ddots & \ddots & \ddots & & \\
\ddots & H_{0}(\overline{\boldsymbol{k}}) & H_{z 1}^{\dagger}(\overline{\boldsymbol{k}}) & H_{z 2}^{\dagger} & 0 & \\
\ddots & H_{z 1}(\overline{\boldsymbol{k}}) & H_{0}(\overline{\boldsymbol{k}}) & H_{z 1}^{\dagger}(\overline{\boldsymbol{k}}) & H_{z 2}^{\dagger} & \ddots \\
\ddots & H_{z 2} & H_{z 1}(\overline{\boldsymbol{k}}) & H_{0}(\overline{\boldsymbol{k}}) & H_{z 1}^{\dagger}(\overline{\boldsymbol{k}}) & \ddots \\
& 0 & H_{z 2} & H_{z 1}(\overline{\boldsymbol{k}}) & H_{0}(\overline{\boldsymbol{k}}) & \ddots \\
& & \ddots & \ddots & \ddots & \ddots
\end{array}\right) .
$$

Here,

$$
H_{0}(\overline{\boldsymbol{k}})=-\sum_{j=x, y} \tau_{x} \sigma_{j} \sin k_{j}+\frac{1}{4}\left(\sigma_{y}+\sigma_{z}\right)+\frac{1}{2} \tau_{z}
$$

is a single-layer Hamiltonian, and

$$
\begin{aligned}
H_{z 1}(\overline{\boldsymbol{k}}) & =\left(-1+\frac{1}{2} \sum_{j=x, y} \cos k_{j}\right) \tau_{z}-\frac{i}{2} \tau_{x} \sigma_{z}, \\
H_{z 2} & =\frac{1}{4} \tau_{z}
\end{aligned}
$$

represents the interlayer coupling. Since the inversion operation is expressed as $\mathcal{P}=\tau_{z}$, the product of the inversion parity of the single-layer Hamiltonian is unity, meaning that a single layer of this model has an even value of the Chern number. In fact, the single layer is a trivial insulator. This example shows that a single layer of a HOTI is not necessarily 


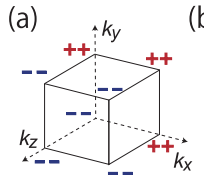

$(b-1)$

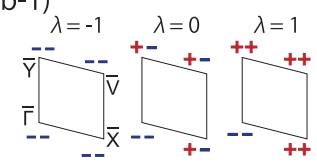

$(b-2)$

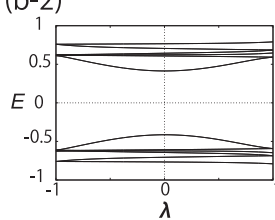

$(b-3)$

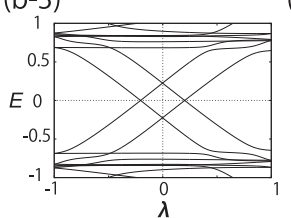

$(\mathrm{c}-3)$

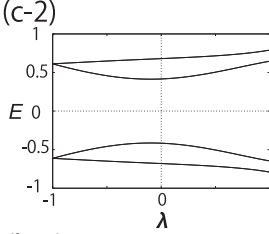

(b-5)
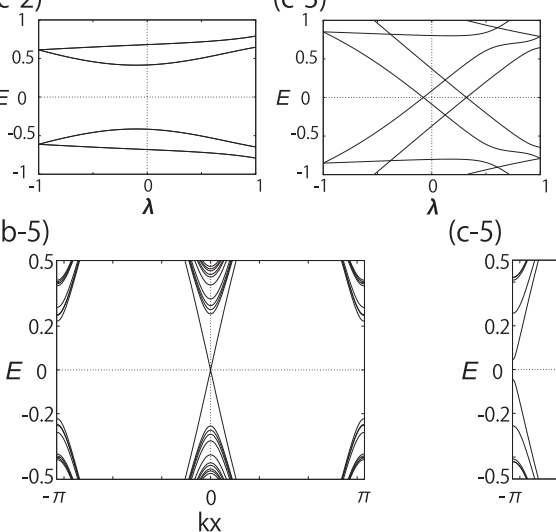

(c-5)

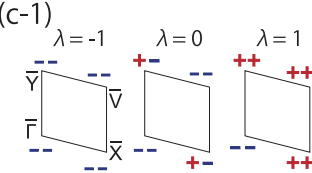

$(b-4)$

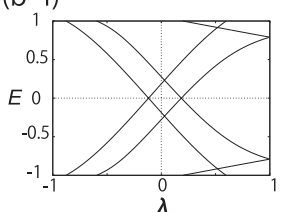

$(\mathrm{c}-4)$
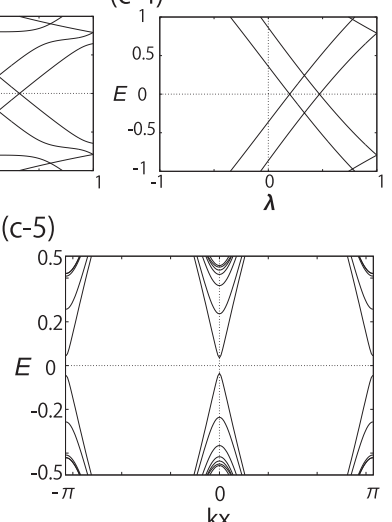

FIG. 10. Numerical calculations for the model Eq. (31) with (b) $L_{z}=12$ and (c) $L_{z}=4$. (a) Inversion parities at TRIM. (b-1), (c-1) Change of the inversion parities at 2D TRIM. In every case, in addition to the state whose parity is shown in (b-1) and (c-1), there are $\frac{\left(L_{z}-2\right) v}{2}$ states with odd parity and $\frac{\left(L_{z}-2\right) v}{2}+2$ states with even parity, and they are not shown in the figure for simplicity. (b-2), (b-3), (b-4), (c-2), (c-3), (c-4) Spectral flow at (b-2), (c-2) $\left(k_{x}, k_{y}\right)=(0,0)=\bar{\Gamma}$, (b-3), (c-3) $(\pi, 0)=\bar{X}$ and $(0, \pi)=\bar{Y},(\mathrm{~b}-4),(\mathrm{c}-4)(\pi, \pi)=\bar{V}$. The spectral flow (b-4) and (c-4) are topologically different. (b-5), (c-5) Hinge energy spectrum. We impose open boundary conditions in $y$ and $z$ direction and set $L_{y}=12$.

a Chern insulator, and the layer-reduction approach does not work straightforwardly in this model.

While the layer-reduction approach does not work in the model Eq. (31), our cutting approach works well. From the numerically calculated spectral flow, we confirm that the slab system is a Chern insulator for a sufficiently large number of layers $L_{z}$. For example, when $L_{z}=12$, the spectral flow is shown in Figs. 10(b-2)-10(b-4). We conclude that the product of the inversion parities at TRIM for the slab system $(\lambda=0)$ is $(-1)$ [see Fig. 10(b-1)]. This means that the slab system has an odd Chern number. We note that in our cutting approach, it is assumed that the bound states at the surfaces at $\lambda= \pm M$ are well localized at the surfaces, meaning that the number of layers $L$ in a slab is much larger than the penetration depth of the surface bound states. Thus our theory does not apply to thin slabs. Indeed in the present model, for small $L_{z}$, such as $L_{z}=4$, the spectral flow and the resulting topological phases are different. In this case, the spectral flow is not symmetric with respect to the transformation $\lambda \rightarrow-\lambda$ as shown in Figs. 10(c-2)-10(c-4). As a result, the product of the inversion parities at TRIM is +1 for the slab system [see Fig. 10(c-1)], and the Chern number is $0(\bmod 2)$.
We show that these differences between a thick slab and a thin slab are reflected in existence or absence of the gapless $1 \mathrm{D}$ states at hinges. When the number of the layer is $L_{z}=12$, there is a chiral 1D mode at $k_{x}=0$ along the hinges as shown in Fig. 10(b-5). However, when $L_{z}=4$, there is no gapless state as shown in Fig. 10(c-5). From these results, we conclude that whether the slab system is Chern insulator or not depends on the thickness of the slab $L_{z}$ for this model. If we simply reducing the number of layers, then the slab system shows a topological phase transition, and becomes a trivial insulator. Therefore, the simple layer-reduction approach does not work for this model. However, our cutting approach applies to general systems, and shows that a $2 \mathrm{D}$ slab of a $3 \mathrm{D}$ HOTI is a Chern insulator, as long as the thickness of the slab is thick enough.

\section{BULK-EDGE AND BULK-HINGE CORRESPONDENCE IN CLASS AII}

In this section, we extend the results of the previous sections to spinful systems with time-reversal symmetry, i.e., class AII systems. In class AII systems, due to the time-reversal symmetry, all energy eigenstates are Kramersdegenerate. Therefore, the number of occupied states $N$ should be an even number. Moreover, since the inversion operator $\mathcal{P}$ commutes with the time-reversal operator $\Theta$, all the inversion eigenstate $|\psi\rangle$ and its Kramers partner $\Theta|\psi\rangle$ have the same parity. This means that the number of occupied states with odd parity is always an even number. Therefore, $N_{-}$and $n_{-}\left(\Gamma_{j}\right)$ should be even. Here, we define $M, M_{-}$ and $m_{-}\left(\Gamma_{j}\right)$ as halves of $N, N_{-}$, and $n_{-}\left(\Gamma_{j}\right)$, respectively, i.e.,

$$
M=\frac{N}{2}, \quad M_{-}=\frac{N_{-}}{2}, \quad m_{-}\left(\Gamma_{j}\right)=\frac{n_{-}\left(\Gamma_{j}\right)}{2} .
$$

By replacing $N, N_{-}$, and $n_{-}$in the previous sections with $M, M_{-}$, and $m_{-}$, respectively, we obtain an extension of our theory to class AII systems. We note that $M$ is equal to the total number of occupied Kramers pairs of states. Likewise, $M_{-}$is equal to the number of occupied Kramers pairs of states with odd parity, and $m_{-}\left(\Gamma_{j}\right)$ is the number of occupied Kramers pairs of states with odd parity at a TRIM $\Gamma_{j}$. We also note that one- and two-dimensional cases are already discussed in the previous study [48]. However, for convenience of the readers, we rewrite these discussions in the previous study in our notation.

\section{A. 1D}

By replacing $N_{-}$and $n_{-}$in Eq. (10) with $M_{-}$and $m_{-}$, respectively, we obtain the following relation:

$$
\begin{aligned}
M_{\mathrm{OBC}} & =M_{\mathrm{PBC}}+m_{-}(0)-m_{-}(\pi)+2\left[M_{-}\right]_{\lambda=1}^{\lambda=0}, \\
& \equiv M_{\mathrm{PBC}}+m_{-}(0)-m_{-}(\pi) \quad(\bmod 2) .
\end{aligned}
$$

This means that the number of occupied Kramers pairs of states at $\lambda=0$ and $\lambda=1$ differ by $1(\bmod 2)$ if $m_{-}(0)-$ $m_{-}(\pi) \equiv 1(\bmod 2)$. 


\section{B. 2D}

By replacing $N$ and $n_{-}$in Eq. (14) with $M$ and $m_{-}$, respectively, we obtain the following relation:

$$
\left.M_{0}\right|_{\lambda=0}-\left.M_{\pi}\right|_{\lambda=0} \equiv \sum_{\Gamma_{j} \in \mathrm{TRIM}} m_{-}\left(\Gamma_{j}\right) \quad(\bmod 2) .
$$

This means that if the right-hand side of Eq. (39) is $1 \bmod 2$, the number of occupied Kramers pairs of states for $\lambda=0$ at $k_{\|}=0$ is different from that at $k_{\|}=\pi$ [48]. To compensate the difference, there should be an odd number of helical edge modes between $k_{\|}=0$ and $k_{\|}=\pi$. We note that this result is consistent with the well-known Fu-Kane formula [54], which shows that the right-hand side of Eq. (39) is equal to the $Z_{2}$ topological invariant of the system.

\section{3D}

By replacing $N$ and $n_{-}$in Eq. (24) with $M$ and $m_{-}$, respectively, we obtain the following relation:

$$
\begin{aligned}
\left.\sum_{\overline{\Gamma_{j}}} \tilde{m}_{-}\left(\overline{\Gamma_{j}}\right)\right|_{\lambda=0} & =\left.2 M\right|_{\lambda=0}-v+\frac{1}{2}\left(-\kappa_{1}\right) \\
& \equiv \frac{1}{2}\left(-\kappa_{1}\right) \quad(\bmod 2) .
\end{aligned}
$$

Here, $\kappa_{1}$ is defined as follows [52]:

$$
\begin{aligned}
\kappa_{1} & =\sum_{\Gamma_{j}: \text { TRIM }}\left[n_{+}\left(\Gamma_{j}\right)-n_{-}\left(\Gamma_{j}\right)\right] / 4 \\
& \equiv-\sum_{\Gamma_{j}: \text { TRIM }} n_{-}\left(\Gamma_{j}\right) / 2 \quad(\bmod 4) \\
& \equiv-\sum_{\Gamma_{j}: \text { TRIM }} m_{-}\left(\Gamma_{j}\right)(\bmod 4) .
\end{aligned}
$$

The left-hand side of Eq. (40) is equal to the $Z_{2}$ topological invariant of the system in a slab geometry [54]. Therefore, if $\kappa_{1}=2(\bmod 4)$, then the system in a slab geometry is a quantum spin Hall insulator. It means that if we make the system to be open also along $x_{2}$ and $x_{3}$ directions, then this system supports helical edge modes with the number of helical modes being an odd number. As in the previous section, these "edge" modes can be on surfaces or on hinges. If we further assume that all the surfaces are gapped, then these helical edge modes are at the hinges. This gives a proof of the bulk-hinge correspondence in inversion-symmetric 3D class AII systems $[33,37,38]$.

\section{BULK-BOUNDARY CORRESPONDENCE IN EXCEPTIONAL CASES}

Here, we show the exceptions to the previous sections and propose modified bulk-boundary correspondences for them. In Sec. II, we begin with a periodic chain consisting of an integer number of unit cells, and cut the chain at an inversion center. From this construction, we see that not every open chain can be treated within our scenario. For example, the open chain in Fig. 11(a-1), where blue and red balls represent different atoms, is outside of our theory because the chain is cut not at an inversion center. The open chain in Fig. 11(a-2) (a-1)

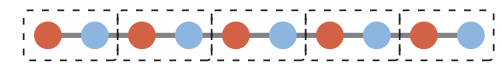

(a-2)

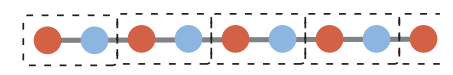

(b)

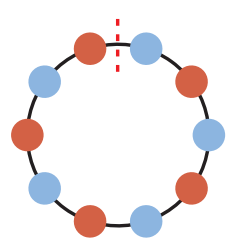

FIG. 11. Examples of the chains which cannot be treated within our scenario with (a-1), (a-2) open boundary conditions, and (b) periodic boundary conditions. Colored balls represent the atomic sites. The red balls and the blue balls are located at distinct inversion centers. (a-1) is obtained by cutting the periodic chain in (b), not at an inversion center. (a-2) cannot be constructed from the periodic chain in (b), because the number of red balls and that of blue balls are different in (a-2).

is also outside of our theory because it cannot be constructed from a periodic chain. Therefore, the periodic chain Fig. 11(b) cannot be treated within our scenario. Since the discussions in Secs. III-V are based on the results in Sec. II, we also need to reconsider the $2 \mathrm{D}$ and $3 \mathrm{D}$ cases.

In this section, we show that the $1 \mathrm{D}$ conventional bulk-edge correspondence does not hold in some models, which are exceptions to the discussion in Sec. II. In such cases, we propose modified 1D bulk-edge correspondence [46]. We also study the exceptional cases in 2D and 3D systems. As a result, unlike the one-dimensional cases, we find that the 2D bulkedge correspondence and the 3D bulk-hinge correspondence hold for any systems.

\section{A. Breakdown of the 1D conventional bulk-edge correspondence}

The fundamental reason that models like Fig. 11 cannot be treated within our scenario is that conventional bulk-edge correspondence, i.e., the correspondence between the bulk inversion parities and the fractional surface charges, breaks down in the model, as we explain in this subsection.

Here, we show an example in which the conventional bulkedge correspondence loses its meaning. This system consists of two sites located at two distinct inversion centers with potential $\pm v$ (see Fig. 12). It is described by the following Hamiltonian $\mathcal{H}(\boldsymbol{k})$ with inversion symmetry $\mathcal{P}_{A}(\boldsymbol{k})$ [55,56]:

$$
\mathcal{H}(\boldsymbol{k})=\left(\begin{array}{cc}
v & 0 \\
0 & -v
\end{array}\right), \quad \mathcal{P}_{A}(\boldsymbol{k})=\left(\begin{array}{cc}
1 & 0 \\
0 & e^{-i k}
\end{array}\right) .
$$

If we set the Fermi level as $E_{F}=0$, then the system is an atomic insulator when $v \neq 0$. When $v<0$, electrons are localized at the A site. However, when $v>0$, electrons are (a)

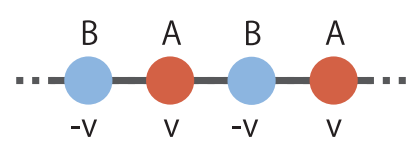

$(b-1)$

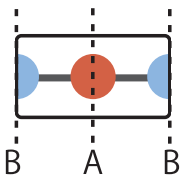

$(\mathrm{b}-2)$

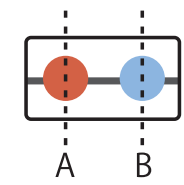

FIG. 12. Sketch of the model Eq. (42). The balls represent atomic sites. (a) The potential is $v(-v)$ at the red (blue) site. (b) Examples of unit cells. The unit cell (b-1) is inversion-symmetric. The unit cell (b-2) is not inversion-symmetric. 
localized at the B site. Also in the open chain, the sign of $v$ determines which site the electrons are localized at. Regardless of whether the open chain is taken as Fig. 11(a-1) or Fig. 11(a-2), the resulting system is an atomic insulator with electrons localized at site A or B. However, $n_{-}(0)-n_{-}(\pi)$ is zero for $v<0$ and minus one for $v>0$, if we take the center of the inversion operation to be the A site. Thus, there is no surface state or fractional surface charge, regardless of the bulk inversion parity. Therefore, the conventional bulk-edge correspondence loses its meaning in this example. This kind of breakdown of the conventional bulk-edge correspondence generally happens if the two distinct inversion centers are occupied by some atomic sites.

This argument shows that the conventional bulk-edge correspondence requires two conditions: (i) the unit cell is inversion symmetric, and (ii) the boundaries of the open chain coincide those of the unit cell. For example, in the open chain in Fig. 11(a-1), we cannot satisfy these two conditions with any choice of a unit cell. The unit cell in Fig. 11(b-1) violates (ii) and that in Fig. 11(b-2) violates (i).

These conditions are also imposed in the recent works on bulk-edge correspondence from the Zak phase [55-58]. In Ref. [55], it is discussed that if (i) or (ii) is violated, even when the Zak phase is $\pi$, no fractional charge appears at the surface. In such cases, more information beyond the bulk Zak phase is required to discuss the surface charge $[56,57]$.

\section{B. 1D modified bulk-edge correspondence}

For the system like Fig. 11, we propose a modified bulkedge correspondence. The modified bulk-edge correspondence is the correspondence between the inversion parities at TRIM in the bulk system and the net parity $\mathcal{I}$ in the open system. The modified bulk-edge correspondence is proposed in Ref. [46] for the system which also exhibit conventional bulk-edge correspondence. We have found that this correspondence holds even when the system does not show the conventional bulk-edge correspondence.

We briefly explain the modified bulk-edge correspondence. The net parity $\mathcal{I}$ is defined as the difference of the number of states with even parity and odd parity:

$$
\mathcal{I}:=N_{+}-N_{-} .
$$

We take the convention different from Ref. [46], and the sign of $\mathcal{I}$ is different. In Ref. [46], for the open system consisting of an integer number of unit cells, they showed the relation between the net parity of the open chain $\left.\mathcal{I}\right|_{\mathrm{OBC}}$ and the bulk inversion parities. In our convention, it is written as follows:

$$
\left.\mathcal{I}\right|_{\mathrm{OBC}}=\left\{\begin{array}{ll}
n_{+}(0)-n_{-}(\pi) & (L: \text { odd }) \\
n_{+}(0)-n_{+}(\pi) & (L: \text { even })
\end{array},\right.
$$

where $L$ is the number of the unit cell. On the right-hand side of Eq. (44), the inversion center is taken to be the center of the unit cell. Since the conventional bulk-edge correspondence also holds in such systems, we can derive Eq. (44) from Eq. (10) (see Appendix E).

For the open chain like Fig. 11(a-2), Eq. (10) does not hold. However, even in such systems, we find that a generalization of Eq. (44) holds. In the generalization of Eq. (44), we choose one of the inversion centers. For example, in the open chain in
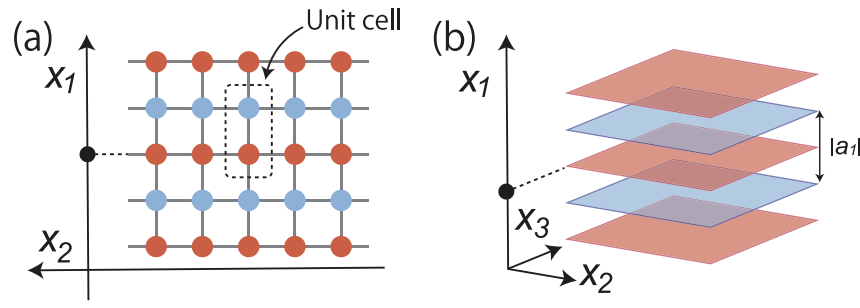

FIG. 13. Examples of exceptions to the bulk-edge correspondence in Secs. III-V. Both examples are finite in the $x_{1}$ direction. The black dots show the position of the inversion center. To preserve the inversion symmetry, both ends of each system are terminated in the same manner. (a) 2D inversion-symmetric system with ribbon geometry. Red and blue ball represent the different sites. (b) $3 \mathrm{D}$ inversion-symmetric layered system with slab geometry. Red and blue planes represent different layers.

Fig. 11(a-2), we take the inversion center to be at the A site. In this case, generalization of Eq. (44) is written as follows:

$$
\left.\mathcal{I}^{(A)}\right|_{\mathrm{OBC}}=n_{+}^{(A)}(0)-n_{-}^{(A)}(\pi) .
$$

Here, the left-hand side is well-defined for the open chain Fig. 11(a-2), because the open chain is inversion-symmetric with respect to the central A site. The detail of the proof is shown in Appendix F.

Finally, we briefly show that Eq. (45) is applicable to the model Eq. (42) with an open chain given in Fig. 11(a-2). When $v<0$, electrons are localized at site $\mathrm{A}$. In this case, the right-hand side is equal to one, because $n_{+}^{(A)}(0)=n_{+}^{(A)}(\pi)=1$ and $n_{-}^{(A)}(0)=n_{-}^{(A)}(\pi)=0$. The left-hand side is also equal to 1 . That is because one $s$-like orbital exist at the inversion center, and other orbitals are not inversion-symmetric and paired by the inversion operation $\mathcal{P}_{A}$. When $v>0$, electrons are localized at site B. In this case, the right-hand side is 0 , because $n_{+}^{(A)}(0)=n_{-}^{(A)}(\pi)=1$ and $n_{-}^{(A)}(0)=n_{+}^{(A)}(\pi)=0$. The left-hand side is also 0 . That is because all states are paired by the inversion operation $\mathcal{P}_{A}$. Therefore, as long as the system is insulating, i.e., $v \neq 0$, Eq. (45) holds.

\section{2D and 3D cases}

Similar to the one-dimensional cases, in 2D and 3D systems, we have some exceptions to the Secs. III-V. We show some examples in Fig. 13. These systems are inversionsymmetric, and cannot be constructed from an integer number of unit cells.

For example, Fig. 13(a) shows a lattice structure of a 2D toy model [55], which cannot be treated in Sec. III. This system is finite in the $x_{1}$ direction, and infinite in the $x_{2}$ direction. If we fix the wave number of the $x_{2}$ direction to 0 or $\pi$, i.e., $k_{2}=0$ or $k_{2}=\pi$, then the system can be considered as a 1D inversion-symmetric system like Fig. 11(a-2). Then, we can apply the modified bulk-edge correspondence, Eq. (45). Similarly, Fig. 13(b) shows a layered system composed of two different layers. For example, the antiferromagnetic Chern insulator has this kind of structure [42,52]. In this system, if we fix $k_{2}$ and $k_{3}$ to 0 or $\pi$, then the system can be considered as a 1D system like Fig. 11(a-2). Again, we can apply Eq. (45). 


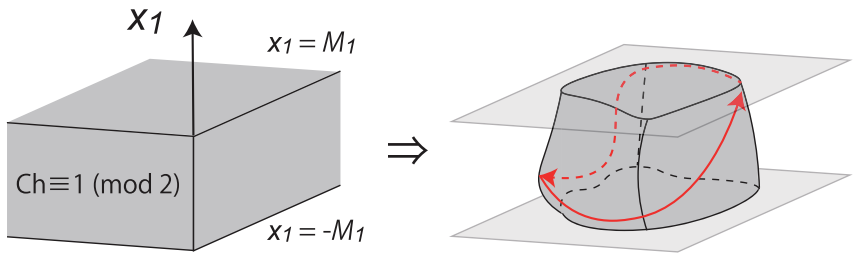

FIG. 14. Conceptual figure of the boundary geometry and the gapless 1D states of the 3D inversion-symmetric HOTI. Red lines represent the gapless hinge states. As long as an infinite slab with surfaces at $x_{1}= \pm M_{1}$ preserves the inversion symmetry and the slab thickness $\left(=2 M_{1}+1\right)$ is thick enough, gapless hinge states appear on the side surfaces, even though the side surfaces are uneven or bent.

As we show in Appendix G, we can derive the 2D bulkedge correspondence and the 3D bulk-hinge correspondence by applying Eq. (45) to 1D subspaces in such systems. As a result, the bulk-boundary correspondence discussed in Secs. III-V also holds in these exceptional cases, and we obtain extended formulas, Eqs. (G4) and (G8).

We note that this kind of exceptions can be seen also in real materials. The antiferromagnetic (AFM) $\mathrm{MnBi}_{2} \mathrm{Te}_{4}$ is one of the examples $[59,60]$. Its unit cell consists of two layers with opposite signs of the spin moments. This is the same as Fig. 13(b), and we can apply our extended formula, Eq. (G8). Since $\mu_{1}=2$ in the AFM $\mathrm{MnBi}_{2} \mathrm{Te}_{4}$, our extended formula tells us that the slab system with an odd number of layers have nonzero Chern number. This is consistent with the Ref. [60].

\section{CONCLUSION AND DISCUSSION}

In the present paper, we studied the bulk-edge and bulkhinge correspondences in inversion-symmetric insulators. We used a cutting procedure, and study the spectral flow in the cutting process. In one- and two-dimensional centrosymmetric systems, we showed that the proof of the bulk-edge correspondence is simplified by introducing a cutting procedure. For a three-dimensional centrosymmetric system, we proved the bulk-hinge correspondence by considering the spectral flow in the cutting process. Unlike the previous approach for the explanation of the bulk-hinge correspondence, our proof is applicable to any tight-binding model with inversion symmetry. We also confirmed this by model calculations, and showed that the spectral flow is consistent with the theoretical calculation.

One of the advantages of our method is its generality. Our method is applicable to any tight-binding model with inversion symmetry. In the main text, we have only considered systems with an odd value of $L$, the number of unit cells in the system. However, as shown in Appendices B and C, our proof can also be applied to systems with even $L$. Moreover, in the main text, we only consider the simple boundary condition. However, as shown in Appendix D, our approach is also applicable to other boundary conditions, as long as $L$ is sufficiently large.

As noted in Sec. VI, Eq. (10) in the main text is not applicable to some open chains like Fig. 11(a-2) and Fig. 13, and the conventional bulk-edge correspondence does not hold. We showed that, the modified bulk-edge correspondence Eq. (45) holds even for such systems by using the modified cutting procedure (see Appendix F). In fact, even for the ordinary systems that also show conventional bulk-edge correspondence, we can derive the modified bulk-edge correspondence Eq. (44) from the conventional one [46] (see Appendix E). From Eqs. (44) and (45), we derive the extended formulas of the 2D bulk-edge correspondence and 3D bulk-hinge correspondence, Eqs. (G4) and (G8) in Appendix G. We emphasize that, though the 1D conventional bulk-edge correspondence does not hold for some systems, 2D bulk-edge correspondence and 3D bulk-hinge correspondence hold for any systems.

In this paper, we studied only the tight-binding models. However, our method is potentially applicable also to real materials. For example, in the first-principle calculation, we can realize cutting procedure by continuously adding an insulating layer. The simplest way is considering a supercell which contains $L_{1}$ unit cells and increasing the size of the supercell while fixing the positions of the atoms. By continuously changing the length of the supercell along $x_{1}$ direction, we can continuously add a vacuum layer at the supercell boundary. In this process, we can realize the cutting process $\lambda=1$ to $\lambda=0$. Therefore, the spectral flow from $\lambda=1$ to $\lambda=0$ can be calculated also in the first-principle calculation.

Our result brings about a new perspective to an interplay between topology and system geometry of the HOTI. In the present paper, we showed that, when we impose an open boundary condition in one direction, a 3D inversionsymmetric HOTI in class A (class AII) becomes a 2D quantum Hall insulator (2D quantum spin Hall insulator). Since the edge modes of these 2D topological insulators appear irrespective of the shape of the boundary, the hinge states of these 3D HOTI always appear irrespective of the boundary shape of the other two directions, $x_{2}$ and $x_{3}$, as shown in Fig. 14. This means that even if the crystal shape of the HOTI does not respect the inversion symmetry, the "hinge" states remain gapless as long as the crystal shape is wide enough in the $x_{2}-x_{3}$ direction (compared with the localization length of the hinge states) as shown in Fig. 14.

\section{ACKNOWLEDGMENTS}

R.T. thanks Motoaki Hirayama for useful comments and discussions. This work was supported by JSPS KAKENHI Grants No. JP18J23289 and No. JP18H03678.

\section{APPENDIX A: UNITARY OPERATOR $U_{x}$ AND APPROXIMATION OF $\mathcal{H}(-\lambda)$}

In this Appendix we show two results which are used in Sec. II: (i) the Hamiltonian with the antiperiodic boundary condition is unitary equivalent to that with the periodic boundary condition with a shifted Bloch wave vector, and (ii) for sufficiently large $L$, the bound states $\left|\psi_{l}(\lambda)\right\rangle$ and $\left|\psi_{l}(-\lambda)\right\rangle$ have opposite parities. Both are derived from the fact that $\mathcal{H}(-\lambda)$ is well-approximated by $U_{x} \mathcal{H}(\lambda) U_{x}^{\dagger}$, where $U_{x}=\exp [i \pi \hat{x} / L]$ is a phase twist operator along $x$.

First, we see how $U_{x} \mathcal{H}(\lambda) U_{x}^{\dagger}$ is related to $\mathcal{H}(-\lambda)$. We consider a one-dimensional periodic system with the coordinate $x$. Let the system size in $x$ direction be $L=2 M+1$ with an integer $M$ measured in the unit of the lattice constant $|\boldsymbol{a}|$. We introduce an open boundary via cutting procedure. We replace 
the hopping amplitudes $t_{j}$ for all the bonds that cross the boundary between $x=-M$ and $x=M$ by $\lambda t_{j}$. For simplicity, at first, we only consider the nearest-neighbor hopping. Then the Hamiltonian is expressed as follows:

$$
\begin{aligned}
\mathcal{H}(\lambda)= & \left(\begin{array}{cccc}
H_{0} & H_{1}^{\dagger} & & \lambda H_{1} \\
H_{1} & H_{0} & \ddots & \\
& \ddots & \ddots & H_{1}^{\dagger} \\
\lambda H_{1}^{\dagger} & & H_{1} & H_{0}
\end{array}\right) \\
= & \sum_{x=-M}^{M} H_{0} \otimes|x\rangle\langle x|+\sum_{x=-M}^{M-1}\left(H_{1} \otimes|x+1\rangle\langle x|+\text { H.c. }\right) \\
& +\left(\lambda H_{1} \otimes|-M\rangle\langle M|+\text { H.c. }\right) .
\end{aligned}
$$

Here, $H_{0}$ and $H_{1}$ are $N_{0} \times N_{0}$ matrices, where $N_{0}$ is the number of states at each unit cell, coming from internal degrees of freedom. $H_{0}$ and $H_{1}$ represent the intra-unit-cell term and the nearest-neighbor hopping term, respectively. $|x\rangle$ is an eigenstate of the position operator $\hat{x}$ at the site $x$. Then $U_{x} \mathcal{H}(\lambda) U_{x}^{\dagger}$ is calculated as follows:

$$
\begin{aligned}
U_{x} \mathcal{H}(\lambda) U_{x}^{\dagger}= & \sum_{x=-M}^{M} H_{0} \otimes|x\rangle\langle x| \\
& +\sum_{x=-M}^{M-1}\left(H_{1} \otimes|x+1\rangle\langle x| e^{i \frac{\pi}{L}(x+1-x)}+\text { H.c. }\right) \\
& +\lambda\left(H_{1} \otimes|-M\rangle\langle M| e^{i \frac{\pi}{L}((-M)-M)}+\text { H.c. }\right) \\
= & \sum_{x=-M}^{M} H_{0} \otimes|x\rangle\langle x| \\
& +\sum_{x=-M}^{M-1}\left(\left(H_{1} e^{i \frac{\pi}{L}}\right) \otimes|x+1\rangle\langle x|+\text { H.c. }\right) \\
& -\lambda\left(\left(H_{1} e^{i \frac{\pi}{L}}\right) \otimes|-M\rangle\langle M|+\text { H.c. }\right) \\
= & \left.\mathcal{H}(-\lambda)\right|_{H_{1} \rightarrow H_{1} e^{i \frac{\pi}{L}} .}
\end{aligned}
$$

More generally, if we include an $m$ th-nearest hopping term $\sum_{x} H_{m} \otimes|x+m\rangle\langle x|+$ H.c., we get

$$
U_{x} \mathcal{H}(\lambda) U_{x}^{\dagger}=\left.\mathcal{H}(-\lambda)\right|_{H_{m} \rightarrow H_{m} e^{i \frac{\pi m}{L}}} .
$$

Therefore, if $m$ is finite and $L$ is sufficiently large, then $U_{x} \mathcal{H}(\lambda) U_{x}^{\dagger}$ is well-approximated by $\mathcal{H}(-\lambda)$.

\section{Antiperiodic Hamiltonian}

Here, we show that the Hamiltonian $\left.\mathcal{H}\right|_{\lambda=-1}$ is unitary equivalent to the periodic Hamiltonian. For this purpose, one introduces the translational operator $T_{x}$ defined as $T_{x}=$ $\sum_{x=-M}^{M-1}|x+1\rangle\langle x|+|-M\rangle\langle M|$. For $\lambda=1$, the Hamiltonian is expressed as follows:

$$
\begin{aligned}
\left.\mathcal{H}\right|_{\lambda=1}= & H_{0} \otimes \sum_{x=-M}^{x=M}|x\rangle\langle x| \\
& +H_{1} \otimes\left(\sum_{x=-M}^{M-1}|x+1\rangle\langle x|+|-M\rangle\langle M|\right)+\text { H.c. } \\
= & H_{0} \otimes \mathbf{1}_{x}+\left[H_{1} \otimes T_{x}+\text { H.c. }\right] .
\end{aligned}
$$

For $\lambda=-1,\left.U_{x} \mathcal{H}\right|_{\lambda=-1} U_{x}^{\dagger}$ is expressed as follows:

$$
\begin{aligned}
\left.U_{x} \mathcal{H}\right|_{\lambda=-1} U_{x}^{\dagger} & H_{0} \otimes \sum_{x=-M}^{x=M}|x\rangle\langle x| \\
& +\left[\left(H_{1} e^{i \frac{\pi}{L}}\right) \otimes\left(\sum_{x=-M}^{M-1}|x+1\rangle\langle x|+|-M\rangle\langle M|\right)+\text { H.c. }\right] \\
= & H_{0} \otimes \mathbf{1}_{x}+\left[\left(H_{1} e^{i \frac{\pi}{L}}\right) \otimes T_{x}+\text { H.c. }\right] \\
= & H_{0} \otimes \mathbf{1}_{x}+\left[H_{1} \otimes \tilde{T}_{x}+\text { H.c. }\right],
\end{aligned}
$$

where $\tilde{T}_{x}=e^{i \frac{\pi}{L}} T_{x}$. Let $e^{i k}, e^{i \tilde{k}}$ be eigenvalues of $T_{x}, \tilde{T}_{x}$, respectively. $k, \tilde{k}$ take values as follows:

$$
\begin{gathered}
k=\frac{2 \pi}{L} m \quad(-M \leqslant m \leqslant M), \\
\tilde{k}=\frac{2 \pi}{L} m+\frac{\pi}{L} \quad(-M \leqslant m \leqslant M) .
\end{gathered}
$$

By comparing Eqs. (A4) and (A5), we conclude that $\left.U_{x} \mathcal{H}\right|_{\lambda=-1} U_{x}^{\dagger}$ is equivalent to the Hamiltonian with the periodic boundary condition with $k$ shifted to $k+\pi / L$. This is natural from the following argument. The case with $\lambda=-1$ corresponds to antiperiodic boundary conditions, leading to the Aharonov-Bohm phase $\pi$ for the whole system. This additional phase $\pi$ appears as an additional term $\pi$ in the formula of $\tilde{k} L$.

\section{Localized states and $U_{x}$}

Here, we show that if $|\psi(\lambda)\rangle$ is an eigenstate of $\mathcal{H}(\lambda)$ with parity $p(= \pm 1)$ localized at the boundaries $x= \pm M$, then $U_{x}|\psi(\lambda)\rangle$ is an eigenstate of $\mathcal{H}(-\lambda)$ with parity $-p$ localized at the boundaries $x= \pm M$. That is because

$$
\begin{aligned}
\mathcal{H}(-\lambda) U_{x}|\psi(\lambda)\rangle & \simeq U_{x} \mathcal{H}(\lambda) U_{x}^{\dagger} U_{x}|\psi(\lambda)\rangle \\
& =U_{x} \mathcal{H}|\psi(\lambda)\rangle \\
& =E(\lambda) U_{x}|\psi(\lambda)\rangle, \\
\mathcal{P} U_{x}|\psi(\lambda)\rangle & =U_{x}^{\dagger} \mathcal{P}|\psi(\lambda)\rangle \\
& =\left(U_{x}^{\dagger}\right)^{2} p U_{x}|\psi(\lambda)\rangle \\
& \simeq(-p) U_{x}|\psi(\lambda)\rangle,
\end{aligned}
$$

where $\simeq$ asymptotically holds true when $L \rightarrow \infty$. Here, we used the relation $\left(U_{x}^{\dagger}\right)^{2}=\exp [-2 i \pi x / L] \simeq-1(x \sim \pm M)$, which holds for localized states at the boundaries $x \simeq \pm M$. From Eqs. (A8) and (A9), we conclude that if $\left|\psi\left(\lambda_{0}\right)\right\rangle$ is a boundary localized energy eigenstate with parity $p$, then there is an eigenstate at $\lambda=-\lambda_{0}$, which have almost the same energy and have opposite parity $-p$. Therefore, if we label the localized states by an integer $l$, then $\left|\psi_{l}(\lambda)\right\rangle$ and $\left|\psi_{l}(-\lambda)\right\rangle$ asymptotically have the same energy for a large system size, and they have opposite parities.

\section{APPENDIX B: ONE-DIMENSIONAL SYSTEM WITH AN EVEN VALUE OF $L$}

In this section, we show that our theory is also applicable to a one-dimensional system with an even value of $L$. There are two different points from the case with an odd value of $L$. The first one is the inversion center for the inversion operation $\mathcal{P}$. 
(a)

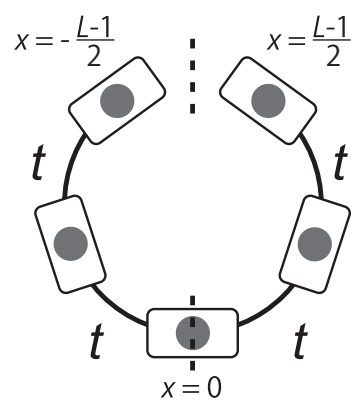

(b)

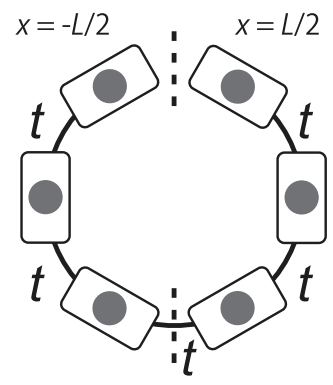

FIG. 15. Difference of the inversion center between (a) the case with odd $L$ and (b) the case with even $L$.

Since the boundary point in the cutting procedure should be the inversion center, the other inversion center $x=0$ is at the center of a unit cell when $L$ is odd, and in between two unit cells when $L$ is even (see Fig. 15). Therefore, the definition of the bulk inversion operator $\mathcal{P}$ is different between odd $L$ and even $L$. Let $\mathcal{P}^{\text {odd }}$ ( $\mathcal{P}^{\text {even }}$ ) denote the bulk inversion operators, with the inversion center being at the center of a unit cell (in the border between two neighboring unit cells). The following equations hold:

$$
\begin{gathered}
\mathcal{P}^{\text {even }}=T_{x} \mathcal{P}^{\text {odd }}, \\
p^{\text {even }}\left(\Gamma_{j}\right)=e^{-i \Gamma_{j}} p^{\text {odd }}\left(\Gamma_{j}\right),
\end{gathered}
$$

where $p^{\text {even }}\left(\Gamma_{j}\right)\left[p^{\text {odd }}\left(\Gamma_{j}\right)\right]$ is an eigenvalue of $\mathcal{P}^{\text {even }}\left(\mathcal{P}^{\text {odd }}\right)$ at $\operatorname{TRIM} \Gamma_{j}=0, \pi$. Therefore,

$$
\begin{aligned}
n_{ \pm}^{\text {even }}(0) & =n_{ \pm}^{\text {odd }}(0), \\
n_{ \pm}^{\text {even }}(\pi) & =n_{\mp}^{\text {odd }}(\pi), \\
= & v-n_{ \pm}^{\text {odd }}(\pi),
\end{aligned}
$$

where $v$ is the number of bulk occupied bands.

The other difference between cases with even $L$ and odd $L$ is the allowed values of $k$. For even $L, k=2 \pi n / L$ can take both 0 and $\pi$. However, $\tilde{k}=2 \pi n / L+\pi / L$ does not take the values of the TRIM. Therefore, $\left.N_{-}^{\text {even }}\right|_{\lambda= \pm 1}$ are calculated as follows:

$$
\begin{gathered}
\left.N_{-}^{\text {even }}\right|_{\lambda=1}=\frac{(L-2) \nu}{2}+n_{-}^{\text {even }}(0)+n_{-}^{\text {even }}(\pi), \\
\left.N_{-}^{\text {even }}\right|_{\lambda=-1}=\frac{L v}{2} .
\end{gathered}
$$

Therefore,

$$
\left.N_{-}^{\text {even }}\right|_{\lambda=1}-\left.N_{-}^{\text {even }}\right|_{\lambda=-1}=-v+n_{-}^{\text {even }}(0)+n_{-}^{\text {even }}(\pi) .
$$

By using this result, the difference between $\left.N\right|_{\lambda=1}$ and $\left.N\right|_{\lambda=0}$ is calculated as follows:

$$
\begin{aligned}
\left.N\right|_{\lambda=1}-\left.N\right|_{\lambda=0}=[N]_{\lambda=0}^{\lambda=1} & \\
& =\left[N_{+}^{\text {even }}\right]_{\lambda=0}^{\lambda=1}+\left[N_{-}^{\text {even }}\right]_{\lambda=0}^{\lambda=1} \\
& =\left[N_{-}^{\text {even }}\right]_{\lambda=0}^{\lambda=-1}+\left[N_{-}^{\text {even }}\right]_{\lambda=0}^{\lambda=1} \\
& =\left[N_{-}^{\text {even }}\right]_{\lambda=1}^{\lambda=-1}+2\left[N_{-}^{\text {even }}\right]_{\lambda=0}^{\lambda=1} \\
& =-\left(-v+n_{-}^{\text {even }}(0)+n_{-}^{\text {even }}(\pi)\right)-2\left[N_{-}^{\text {even }}\right]_{\lambda=1}^{\lambda=0},
\end{aligned}
$$

or equivalently,

$$
\begin{aligned}
\left.N\right|_{\lambda=0}= & \left.N\right|_{\lambda=1}+\left(-v+n_{-}^{\text {even }}(0)+n_{-}^{\text {even }}(\pi)\right) \\
& +2\left[N_{-}^{\text {even }}\right]_{\lambda=1}^{\lambda=0} .
\end{aligned}
$$

By taking modulo 2 on both sides of the Eq. (B9), we get the following equation:

$$
\left.\left.N\right|_{\lambda=0} \equiv N\right|_{\lambda=1}+\left[-v+n_{-}^{\text {even }}(0)+n_{-}^{\text {even }}(\pi)\right] \quad(\bmod 2) .
$$

This means that the parity of the number of occupied states at $\lambda=0$ is calculated from the knowledge of $n_{-}^{\text {even }}$.

Here, we show that this result gives the same value of $\left.N\right|_{\lambda=0}$ as the case with odd $L$ in Eq. (1). From Eqs. (B3) and (B4), the second term on the right-hand side of Eq. (B10) is rewritten as follows:

$$
\begin{aligned}
-v+n_{-}^{\text {even }}(0)+n_{-}^{\text {even }}(\pi) & =-v+n_{-}^{\text {odd }}(0)+n_{+}^{\text {odd }}(\pi) \\
& =n_{-}^{\text {odd }}(0)-n_{-}^{\text {odd }}(\pi) .
\end{aligned}
$$

From Eqs. (B10) and (B11), we obtain Eq. (1). This clearly shows that the value of $\left.N\right|_{\lambda=0}$ obtained here is the same as the case with odd $L$, which is physically reasonable.

\section{APPENDIX C: THREE-DIMENSIONAL SYSTEM WITH EVEN $L_{1}$}

In this section, we show that our theory is also applicable to a three-dimensional system with even $L_{1}$. The important point is that $\mu_{1}$ depends on the choice of the inversion center, if and only if $v_{1} \neq 0$. Since the choice of the inversion center depends on the even-oddness of $L_{1}$ as explained in Appendix B, $\mu_{1}$ depends on $L_{1}$. Therefore, the Chern number of the system with open boundary also depends on $L_{1}$ when $v_{1} \neq 0$. As shown below, the $L_{1}$ dependence of the Chern number is physically reasonable. This is the main focus of this section.

First, as in the case of odd $L_{1}$ studied in Sec. IV A, we show that the Chern number $(\bmod 2)$ of the system with open boundary condition is equal to the bulk topological number $\mu_{1}$. We write $n_{-}^{\text {even }}$ as $n_{-}^{\text {ev }}$ in this section. From Eqs. (B5) and (B9), the following relation holds:

$$
\begin{aligned}
& \left.\tilde{n}_{-}^{\mathrm{ev}}\left(\overline{\Gamma_{j}}\right)\right|_{\lambda=1}=\frac{\left(L_{1}-2\right) v}{2}+n_{-}^{\mathrm{ev}}\left(0, \overline{\Gamma_{j}}\right)+n_{-}^{\mathrm{ev}}\left(\pi, \overline{\Gamma_{j}}\right), \\
& {\left[\tilde{n}_{-}^{\mathrm{ev}}\left(\overline{\Gamma_{j}}\right)\right]_{\lambda=1}^{\lambda=0}=\frac{\left[N_{\overline{\Gamma_{j}}}\right]_{\lambda=1}^{\lambda=0}}{2}+\frac{v-n_{-}^{\mathrm{ev}}\left(0, \overline{\Gamma_{j}}\right)-n_{-}^{\mathrm{ev}}\left(\pi, \overline{\Gamma_{j}}\right)}{2} .}
\end{aligned}
$$

By adding Eq. (C1) and Eq. (C2), we obtain the following equation:

$$
\left.\tilde{n}_{-}^{\mathrm{ev}}\left(\overline{\Gamma_{j}}\right)\right|_{\lambda=0}=\left.\frac{1}{2} N_{\overline{\Gamma_{j}}}\right|_{\lambda=0}-\frac{v}{2}+\frac{n_{-}^{\mathrm{ev}}\left(0, \overline{\Gamma_{j}}\right)+n_{-}^{\mathrm{ev}}\left(\pi, \overline{\Gamma_{j}}\right)}{2},
$$

where we used the relation $\left.N_{\overline{\Gamma_{j}}}\right|_{\lambda=0}=L \nu$. By taking the summation of Eq. (C3) over the $2 \mathrm{D} \operatorname{TRIM} \overline{\Gamma_{j}}$, we obtain the 
following relation:

$$
\begin{aligned}
\left.\sum_{\overline{\Gamma_{j}}} \tilde{n}_{-}^{\mathrm{ev}}\left(\overline{\Gamma_{j}}\right)\right|_{\lambda=0}= & \left.\frac{1}{2} \sum_{\overline{\Gamma_{j}}} N_{\overline{\Gamma_{j}}}\right|_{\lambda=0}-2 v \\
& +\sum_{\overline{\Gamma_{j}}} \frac{n_{-}^{\mathrm{ev}}\left(0, \overline{\Gamma_{j}}\right)+n_{-}^{\mathrm{ev}}\left(\pi, \overline{\Gamma_{j}}\right)}{2} \\
\equiv & \frac{1}{2} \sum_{\Gamma_{j}} n_{-}^{\mathrm{ev}}\left(\Gamma_{j}\right) \quad(\bmod 2) .
\end{aligned}
$$

Equation (C5) is completely the same form with Eq. (18), except for the definitions of $\tilde{n}_{-}$and $n_{-}$: the parity is defined by $\mathcal{P}^{\text {odd }}$ in Eq. (18) and by $\mathcal{P}^{\text {even }}$ in Eq. (C5). Here, $\mathcal{P}^{\text {odd }}$ and $\mathcal{P}^{\text {even }}$ are defined as in Appendix B. Therefore, from Eqs. (B3) and (B4), Eqs. (C4) and (C5) are rewritten in terms of as follows:

$$
\begin{aligned}
\left.\sum_{\overline{\Gamma_{j}}} \tilde{n}_{-}^{\mathrm{ev}}\left(\overline{\Gamma_{j}}\right)\right|_{\lambda=0}= & \frac{1}{2} \sum_{\overline{\Gamma_{j}}} N_{\overline{\Gamma_{j}}} \mid \lambda=0 \\
& +\sum_{\overline{\Gamma_{j}}} \frac{n_{-}^{\text {odd }}\left(0, \overline{\Gamma_{j}}\right)-n_{-}^{\text {odd }}\left(\pi, \overline{\Gamma_{j}}\right)}{2} \\
\equiv & \frac{1}{2} \sum_{\Gamma_{j}} n_{-}^{\text {odd }}\left(\Gamma_{j}\right)-v_{1} \quad(\bmod 2) .
\end{aligned}
$$

From Eqs. (18) and (C7), we have the following relation:

$$
\begin{gathered}
\left.\left.\sum_{\overline{\Gamma_{j}}} \tilde{n}_{-}^{\text {ev }}\left(\overline{\Gamma_{j}}\right)\right|_{\lambda=0} \equiv \sum_{\overline{\Gamma_{j}}} \tilde{n}_{-}^{\text {odd }}\left(\overline{\Gamma_{j}}\right)\right|_{\lambda=0}-v_{1} \quad(\bmod 2), \\
\left.\left.(\text { Chern })^{\text {even }}\right|_{\lambda=0} \equiv(\text { Chern })^{\text {odd }}\right|_{\lambda=0}-v_{1} \quad(\bmod 2) .
\end{gathered}
$$

Therefore, if $v_{1} \neq 0$, then the Chern number of the open 2D system depends on the even-oddness of $L_{1}$.

Here, we give an illustrative example, to show that the $L_{1}$ dependence of the Chern number is physically reasonable. Let us consider a system formed by stacking 2D Chern insulators with Chern number +1 . We assume that the stacking direction is the $x_{1}$ direction. In Fig. 16, we illustrate the stacked Chern insulators with (a) an odd number of layers and (b) an even number of layers. For simplicity, we assume the interlayer hopping term to be 0 . Then the 2D Chern number of the system perpendicular to $x_{1}$ axis is simply calculated as a sum of the Chern number of each layer, and therefore it is equal to the thickness of the layer $L_{1}$. In this case, obviously, the Chern number $(\bmod 2)$ is equal to $L_{1}$ in accordance with Eq. (C9).

Next, we additionally assume that the Chern insulator preserves inversion symmetry. Then, we can calculate the Chern number (mod 2) from the knowledge of the bulk inversion parity, via Eq. (18) or Eq. (C5). As an example, we consider the case that the parity eigenvalues of the Chern insulator are as shown in Fig. $16(\mathrm{c})$, i.e., $n_{-}(\bar{\Gamma})=1$ and $n_{-}\left(\overline{\Gamma_{j}}\right)=0$ $\left(\overline{\Gamma_{j}}=\bar{Y}, \bar{Z}, \bar{T}\right)$. When we stack the Chern insulator with no interlayer hopping, the bulk parity of the stacked 3D system at TRIM are directly calculated from the 2D bulk parity of the Chern insulator layer. The results depend on the evenoddness of the number of layers $L_{1}$, and shown in Figs. 16(d) and 16(e). The $L_{1}$ dependence comes from the difference of the inversion center as shown in Figs. 16(a) and 16(b). From Eqs. (18) and (C5), the Chern number $(\bmod 2)$ of the (a)

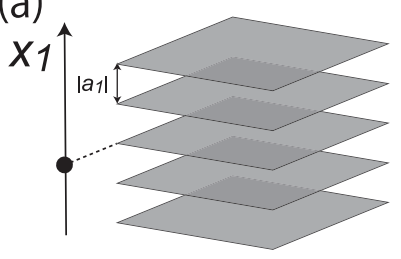

(b)
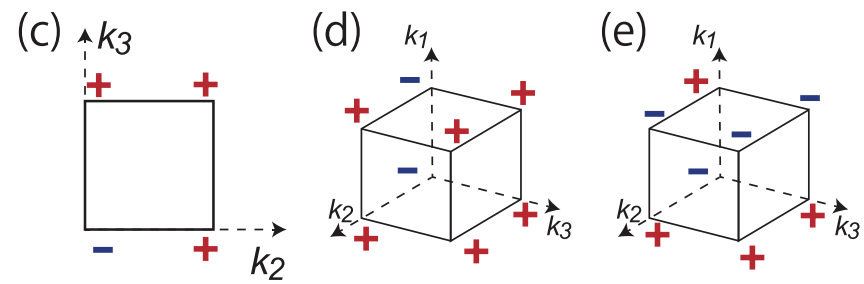

FIG. 16. Conceptual figures of a stacking of $L_{1}$ layers of Chern insulators with (a) odd $L_{1}$ and (b) even $L_{1}$. Gray planes mean Chern insulator layers stacked along the $x_{1}$ direction. In (a), the inversion center is within one of the Chern insulator layer. In (b), the inversion center is in between the two layers. (c) An example of inversion parities of the Chern insulator layer. (d), (e) The inversion parities of the stacked Chern insulators with (d) odd $L_{1}$ and (e) even $L_{1}$. The difference of the parities comes from the difference of the inversion center.

open system is calculated as $\left.(\text { Chern })^{\text {odd }}\right|_{\lambda=0} \equiv 1(\bmod 2)$ and $\left.(\text { Chern })^{\text {even }}\right|_{\lambda=0} \equiv 2 \equiv 0(\bmod 2)$, respectively. These results are consistent with the fact that the Chern number is equal to $L_{1}$.

\section{APPENDIX D: BOUNDARY TERM}

In the main text, we only consider the simple open boundary condition. Here, we show that our results can also be applied to cases with other boundary conditions. We consider the boundary term defined as follows:

$$
\begin{aligned}
& \mathcal{H}_{S}=\left(\begin{array}{lllll}
\mathcal{H}^{A} & & & & \\
& \text { 0 } & & & \\
& & \ddots & & \\
& & & \mathbf{0} & \\
& & & & \mathcal{H}^{B}
\end{array}\right) \\
& =\sum_{x, x^{\prime}=0}^{l_{A}} \mathcal{H}_{x, x^{\prime}}^{A}|x\rangle\left\langle x^{\prime}\left|+\sum_{x, x^{\prime}=0}^{l_{B}} \mathcal{H}_{L-x, L-x^{\prime}}^{B}\right| L-x\right\rangle\left\langle L-x^{\prime}\right|,
\end{aligned}
$$

where $l_{A}, l_{B}$ are small finite integers compared with the system size $L$. $\mathcal{H}_{x, x^{\prime}}^{A}$ and $\mathcal{H}_{L-x, L-x^{\prime}}^{B}$ are $N_{0} \times N_{0}$ matrices which represent the change of the boundary condition. We assume that the boundary term does not break the inversion symmetry: $\mathcal{P} \mathcal{H}_{S} \mathcal{P}^{\dagger}=\mathcal{H}_{S}$. We add this term to the Hamiltonian with the simple boundary condition defined in Eq. (A1): $\mathcal{H}^{\text {basic }}(\lambda) \stackrel{\text { def }}{=}$ $\mathcal{H}(\lambda)$. Then the Hamiltonian with the general boundary condition $\mathcal{H}^{\text {general }}(\lambda)$ is defined as follows:

$$
\mathcal{H}^{\text {general }}(\lambda)=\mathcal{H}^{\text {basic }}(\lambda)+\left(1-\lambda^{2}\right) \mathcal{H}_{S} .
$$


The additional term is added with a factor $1-\lambda^{2}$ to guarantee that it vanishes at $\lambda= \pm 1$. In this section, we show that the additional term $\left(1-\lambda^{2}\right) \mathcal{H}_{S}$ does not change the results in the previous sections.

First, the Hamiltonian $\mathcal{H}^{\text {general }}(\lambda)$ at $\lambda= \pm 1$ are completely the same with $\mathcal{H}^{\text {basic }}(\lambda)$. Therefore, Eq. (A5) holds even when $\mathcal{H}(\lambda)$ is replaced by $\mathcal{H}^{\text {general }}(\lambda)$. Next, we show that $U_{x} \mathcal{H}_{S} U_{x}^{\dagger}$ is well-approximated by $\mathcal{H}_{S}$ :

$$
\begin{aligned}
U_{x} \mathcal{H}_{S} U_{x}^{\dagger}= & \sum_{x, x^{\prime}=0}^{l_{A}} \mathcal{H}_{x, x^{\prime}}^{A} e^{\frac{i \pi}{L}\left(x-x^{\prime}\right)}|x\rangle\left\langle x^{\prime}\right| \\
& +\sum_{x, x^{\prime}=0}^{l_{B}} \mathcal{H}_{L-x, L-x^{\prime}}^{B} e^{-\frac{i \pi}{L}\left(x-x^{\prime}\right)}|L-x\rangle\left\langle L-x^{\prime}\right| \\
\simeq & \mathcal{H}_{S} \quad(L \rightarrow \infty) .
\end{aligned}
$$

Here, we used the fact that $e^{\frac{i \pi}{L}\left(x-x^{\prime}\right)} \rightarrow 1(L \rightarrow \infty)$, since $\mid x-$ $x^{\prime} \mid \leqslant l_{A}, l_{B}$ holds. From Eqs. (A3) and (D3), we obtain the following relation:

$$
\begin{aligned}
U_{x} \mathcal{H}^{\text {general }}(\lambda) U_{x}^{\dagger} & =U_{x} \mathcal{H}^{\text {basic }}(\lambda) U_{x}^{\dagger}+\left(1-\lambda^{2}\right) U_{x} \mathcal{H}_{S} U_{x}^{\dagger} \\
& \simeq \mathcal{H}^{\text {basic }}(-\lambda)+\left(1-(-\lambda)^{2}\right) \mathcal{H}_{S} \\
& =\mathcal{H}^{\text {general }}(-\lambda) .
\end{aligned}
$$

By comparing Eqs. (A8) and (D4), we can see that Eq. (A8) holds even when $\mathcal{H}(\lambda)$ is replaced by $\mathcal{H}^{\text {general }}(\lambda)$.

Combining the above two results, we conclude that our calculation holds true even when $\mathcal{H}(\lambda)$ is replaced by $\mathcal{H}^{\text {general }}(\lambda)$.

\section{APPENDIX E: DERIVATION OF EQ. (44) FROM EQ. (10)}

Here, we focus on a system which shows the conventional bulk-edge correspondence. For such a system, we derive the modified bulk-edge correspondence, Eq. (44), from the conventional one, Eq. (10). We note that $\lambda=0(\lambda=1)$ corresponds to the open boundary condition (the periodic boundary condition), respectively.

First, we consider the case with odd $L$. Since $N=N_{+}+N_{-}$, Eq. (10) is rewritten as follows:

$$
\left.\left(N_{+}-N_{-}\right)\right|_{\mathrm{OBC}}=\left.\left(N-2 N_{-}\right)\right|_{\mathrm{PBC}}+n_{-}(0)-n_{-}(\pi) .
$$

Here, the left-hand side is equal to $\left.\mathcal{I}\right|_{\text {OBC. From Eq. (3) and }}$ $\left.N\right|_{\mathrm{PBC}}=L v$, we obtain the following:

$$
\begin{aligned}
\left.\mathcal{I}\right|_{\text {овС }} & =\left(v-2 n_{-}(0)\right)+n_{-}(0)-n_{-}(\pi) \\
& =n_{+}(0)-n_{-}(\pi) \quad(L: \text { odd }),
\end{aligned}
$$

where we used $v=n_{+}(0)+n_{-}(0)$. This is exactly Eq. (44) with odd $L$.

Next, we consider the case with even $L$. In this case, the explicit form of $\left.N_{-}\right|_{\mathrm{PBC}}$ changes to Eq. (B5) from Eq. (3). Therefore, the first term on the right-hand side of Eq. (E1) changes as follows:

$$
\begin{aligned}
\left.\left(N-2 N_{-}\right)\right|_{\mathrm{PBC}} & =2 v-2 n_{-}(0)-2 n_{+}(\pi) \\
& =v-2 n_{-}(0)+n_{-}(\pi)-n_{+}(\pi) .
\end{aligned}
$$
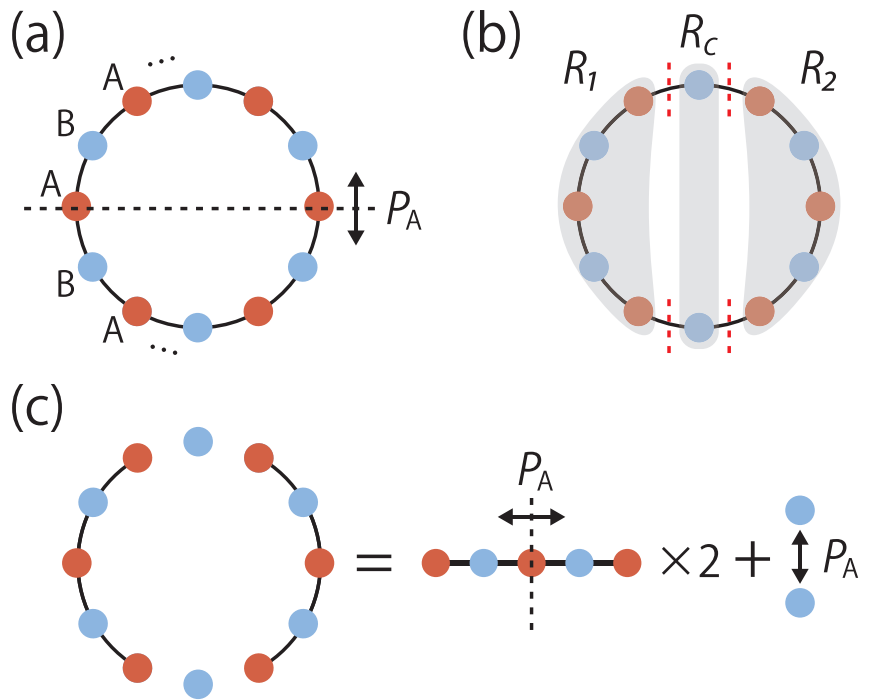

FIG. 17. Conceptual figure of the cutting procedure for deriving Eq. (45). (a) Periodic chain which is invariant under the inversion operation $\mathcal{P}_{A}$. (b) Dividing the chain into three regions, $R_{1}, R_{2}, R_{c}$, and introducing a cutting at the red dashed line. Each region is invariant by $\mathcal{P}_{A} . R_{1}$ and $R_{2}$ have a site which is invariant by $\mathcal{P}_{A}$, but $R_{C}$ does not. (c) After the cutting process, divided regions are considered as a direct sum of the two open chains $\left(R_{1}\right.$ and $\left.R_{2}\right)$ and the remaining part $\left(R_{c}\right)$.

Therefore, Eq. (E2) changes as follows:

$$
\left.\mathcal{I}\right|_{\mathrm{OBC}}=n_{+}(0)-n_{+}(\pi) \quad(L \text { : even }) .
$$

This is exactly Eq. (44) with even $L$.

\section{APPENDIX F: DERIVATION OF EQ. (45)}

In this section, we give a proof of Eq. (45), particularly for the open chain shown in Fig. 11(a-2). We take the inversion center to be at the site A. We consider the periodic chain as shown in Fig. 17(a). We assume that $L=2 M$ ( $M$ : integer), and $M$ is sufficiently large. We divide the system into three regions $R_{1}, R_{2}, R_{c}$ as shown in Fig. 17(b). We assume that $R_{1}$ and $R_{2}$ are invariant by the inversion operation $\mathcal{P}_{A}$, and these regions are sufficiently large. We introduce a cutting through the cutting procedure at the boundary of the regions as shown in Fig. 17(b). When $\lambda=1$, the total system is a periodic chain. When $\lambda=0$, the system is considered as a direct sum of the two inversion-symmetric open chains $\left(R_{1}\right.$ and $\left.R_{2}\right)$ and the remaining parts $\left(R_{c}\right)$ as shown in Fig. 17(c).

First, when $\lambda=1,\left.\mathcal{I}_{\text {total }}^{(A)}\right|_{\lambda=1}$ is calculated from the bulk inversion parities:

$$
\begin{aligned}
\left.\mathcal{I}_{\text {total }}^{(A)}\right|_{\lambda=1} & :=\left.N_{+, \text {total }}^{(A)}\right|_{\lambda=1}-\left.N_{-, \text {total }}^{(A)}\right|_{\lambda=1} \\
& =n_{+}^{(A)}(0)+n_{+}^{(A)}(\pi)-n_{-}^{(A)}(0)-n_{-}^{(A)}(\pi) \\
& =2\left[n_{+}^{(A)}(0)-n_{-}^{(A)}(\pi)\right] .
\end{aligned}
$$

Next, when $\lambda=0$, since the total system is considered as a direct sum of three regions, $\left.\mathcal{I}_{\text {total }}^{(A)}\right|_{\lambda=0}$ is represented as a summation of $\mathcal{I}_{R_{j}}^{(A)}\left(R_{j}=R_{1}, R_{2}, R_{c}\right)$ in each region:

$$
\left.\mathcal{I}_{\text {total }}^{(A)}\right|_{\lambda=0}=\mathcal{I}_{R_{1}}^{(A)}+\mathcal{I}_{R_{2}}^{(A)}+\mathcal{I}_{R_{c}}^{(A)} \text {. }
$$


Since the regions $R_{1}$ and $R_{2}$ are equivalent to the inversion-symmetric open chain, $\mathcal{I}_{R_{1}}^{(A)}=\mathcal{I}_{R_{2}}^{(A)}=\left.\mathcal{I}^{(A)}\right|_{\mathrm{OBC}}$. Here, $\left.\mathcal{I}^{(A)}\right|_{\text {OBC }}$ is a generalization on the left-hand side of Eq. (44), to the $\mathcal{P}_{A}$-symmetric open chain as shown in Fig. 11(a-2). More precisely, $\left.\mathcal{I}^{(A)}\right|_{\mathrm{OBC}}$ is defined as the difference of the number of states with even parity and odd parity in the open chain Fig. 11(a-2):

$$
\left.\mathcal{I}^{(A)}\right|_{\mathrm{OBC}}:=\left.N_{+}^{(A)}\right|_{\text {ОвС }}-\left.N_{-}^{(A)}\right|_{\text {ОвC }} \cdot
$$

Moreover, since there is no inversion-symmetric site in the region $R_{c}, \mathcal{I}_{R_{c}}^{(A)}=0$. Therefore, Eq. (F2) is rewritten as follows:

$$
\left.\mathcal{I}_{\text {total }}^{(A)}\right|_{\lambda=0}=\left.2 \mathcal{I}^{(A)}\right|_{\text {OBC }} \text {. }
$$

Finally, if the two regions $R_{1}$ and $R_{2}$ are sufficiently large, then the boundary of the regions are far from the inversion center of $\mathcal{P}_{A}$. Therefore, the total net inversion parity $\mathcal{I}_{\text {total }}$ is constant in the cutting process:

$$
\left.\mathcal{I}_{\text {total }}\right|_{\lambda=1}=\left.\mathcal{I}_{\text {total }}\right|_{\lambda=0} \text {. }
$$

From Eqs. (F1), (F4), and (F5), we obtain the following relation:

$$
\left.\mathcal{I}^{(A)}\right|_{\mathrm{OBC}}=n_{+}^{(A)}(0)-n_{-}^{(A)}(\pi) .
$$

This is exactly Eq. (45).

\section{APPENDIX G: APPLICATION OF EQS. (44) AND (45) TO 2D AND 3D SYSTEMS}

In this section, we generalize the result of the main text to $2 \mathrm{D}$ and $3 \mathrm{D}$ systems by using Eqs. (44) and (45). Since Eqs. (44) and (45) have almost the same form, in the following, we only show for the case with Eq. (44). The proof for the case with Eq. (45) is obtained by replacing $\mathcal{I}, n_{-}$to $\mathcal{I}^{(A)}, n_{-}^{(A)}$. Moreover, for simplicity, we consider the case with odd $L$.

\section{2D bulk-edge correspondence}

We consider 1D $\mathcal{P}$-invariant subspaces of $\boldsymbol{k}$ space, $k_{2}=$ 0 and $k_{2}=\pi$, which we call $\bar{\Gamma}$ and $\bar{Y}$, respectively. We apply Eq. (44) to these subspaces and obtain the following equations:

$$
\begin{aligned}
\left.\mathcal{I}_{\bar{\Gamma}}\right|_{\mathrm{OBC}} & =n_{+}(\Gamma)-n_{-}(X) \\
& =v-n_{-}(\Gamma)-n_{-}(X), \\
\left.\mathcal{I}_{\bar{Y}}\right|_{\mathrm{OBC}} & =v-n_{-}(Y)-n_{-}(M) .
\end{aligned}
$$

Since $\mathcal{I}=N-2 N_{-}, \mathcal{I}$ and $N$ are equivalent modulo 2 :

$$
\left.\left.\mathcal{I}\right|_{\text {овС }} \equiv N\right|_{\text {овС }} \quad(\bmod 2) \text {. }
$$

By subtracting Eq. (G1) from Eq. (G2), and taking modulo 2, we obtain the following equation:

$$
\begin{aligned}
\left.N_{\bar{Y}}\right|_{\mathrm{OBC}}-\left.N_{\bar{\Gamma}}\right|_{\mathrm{OBC}} & \equiv n_{-}(\Gamma)+n_{-}(X)-n_{-}(Y)-n_{-}(M) \\
& \equiv \sum_{\Gamma_{j} \in \mathrm{TRIM}} n_{-}\left(\Gamma_{j}\right) \quad(\bmod 2) .
\end{aligned}
$$

Equation (G4) is a generalization of the Eq. (14).

\section{3D bulk-hinge correspondence}

We consider 1D $\mathcal{P}$-invariant subspaces of $\boldsymbol{k}$ space. $\left(k_{2}, k_{3}\right)=(0,0),(\pi, 0),(0, \pi),(\pi, \pi)$, which we call $\bar{\Gamma}, \bar{Y}$, $\bar{Z}, \bar{T}$, respectively. We apply Eq. (44) to these subspaces and obtain the following equations:

$$
\begin{aligned}
\left.\mathcal{I}_{\overline{\Gamma_{j}}}\right|_{\mathrm{OBC}} & =n_{+}\left(0, \overline{\Gamma_{j}}\right)-n_{-}\left(\pi, \overline{\Gamma_{j}}\right) \\
& =v-n_{-}\left(0, \overline{\Gamma_{j}}\right)-n_{-}\left(\pi, \overline{\Gamma_{j}}\right),
\end{aligned}
$$

where $\overline{\Gamma_{j}}=\{\bar{\Gamma}, \bar{Y}, \bar{Z}, \bar{T}\}$, and $\left(0, \overline{\Gamma_{j}}\right)$ and $\left(\pi, \overline{\Gamma_{j}}\right)$ are 3D TRIM.

Since $\mathcal{I}=N-2 N_{-},\left.\quad N_{-}\left(\overline{\Gamma_{j}}\right)\right|_{\text {OBC }}$ is represented as follows:

$$
\begin{aligned}
\left.N_{-}\left(\overline{\Gamma_{j}}\right)\right|_{\text {овС }} & =\frac{1}{2}\left(\left.N_{\overline{\Gamma_{j}}}\right|_{\text {овС }}-\left.\mathcal{I}\right|_{\text {ОвС }}\right) \\
& =\frac{1}{2}\left[\left.N_{\overline{\Gamma_{j}}}\right|_{\text {овС }}-v+n_{-}\left(0, \overline{\Gamma_{j}}\right)+n_{-}\left(\pi, \overline{\Gamma_{j}}\right)\right] .
\end{aligned}
$$

In Eq. (18), $\tilde{n}_{-}\left(\overline{\Gamma_{j}}\right)$ is defined as the number of occupied states with odd parity at the 2D TRIM $\overline{\Gamma_{j}}$. From the definition, this is equivalent to the total number of occupied states with odd parity in the $1 \mathrm{D}$ subspace $\boldsymbol{k}=\left(k_{1}, \overline{\Gamma_{j}}\right), N_{-}\left(\overline{\Gamma_{j}}\right)$ :

$$
\tilde{n}_{-}\left(\overline{\Gamma_{j}}\right)=N_{-}\left(\overline{\Gamma_{j}}\right) .
$$

By taking the summation of Eq. (G6) over the 2D TRIM $\overline{\Gamma_{j}}$, and using Eq. (G7), we obtain the following relation:

$$
\begin{aligned}
\left.\sum_{\overline{\Gamma_{j}}} \tilde{n}_{-}\left(\overline{\Gamma_{j}}\right)\right|_{\mathrm{OBC}} & =\left.\frac{1}{2} \sum_{\overline{\Gamma_{j}}} N_{\overline{\Gamma_{j}}}\right|_{\mathrm{OBC}}-2 v+\frac{1}{2} \sum_{\Gamma_{j}} n_{-}\left(\Gamma_{j}\right) \\
& =2 N_{\mathrm{OBC}}-2 v+\frac{1}{2} \sum_{\Gamma_{j}} n_{-}\left(\Gamma_{j}\right) \\
& \equiv \frac{1}{2} \sum_{\Gamma_{j}} n_{-}\left(\Gamma_{j}\right) \equiv \frac{1}{2}\left(-\mu_{1}\right) \quad(\bmod 2),
\end{aligned}
$$

where we used the assumption that the system is insulating under the open-boundary condition; i.e., $\left.N_{\overline{\Gamma_{j}}}\right|_{\text {овC }}=N_{\mathrm{OBC}}$. Equation (G8) is a generalization of the Eq. (18).
[1] M. Z. Hasan and C. L. Kane, Colloquium: Topological insulators, Rev. Mod. Phys. 82, 3045 (2010).

[2] X.-L. Qi and S.-C. Zhang, Topological insulators and superconductors, Rev. Mod. Phys. 83, 1057 (2011).

[3] D. J. Thouless, M. Kohmoto, M. P. Nightingale, and M. den Nijs, Quantized Hall Conductance in a Two-Dimensional Periodic Potential, Phys. Rev. Lett. 49, 405 (1982).
[4] R. B. Laughlin, Quantized Hall conductivity in two dimensions, Phys. Rev. B 23, 5632(R) (1981).

[5] Y. Hatsugai, Chern Number and Edge States in the Integer Quantum Hall Effect, Phys. Rev. Lett. 71, 3697 (1993).

[6] C. L. Kane and E. J. Mele, $Z_{2}$ Topological Order and the Quantum Spin Hall Effect, Phys. Rev. Lett. 95, 146802 (2005). 
[7] C. L. Kane and E. J. Mele, Quantum Spin Hall Effect in Graphene, Phys. Rev. Lett. 95, 226801 (2005).

[8] B. A. Bernevig and S.-C. Zhang, Quantum Spin Hall Effect, Phys. Rev. Lett. 96, 106802 (2006).

[9] J. Zak, Berry's Phase for Energy Bands in Solids, Phys. Rev. Lett. 62, 2747 (1989).

[10] R. D. King-Smith and D. Vanderbilt, Theory of polarization of crystalline solids, Phys. Rev. B 47, 1651(R) (1993).

[11] D. Vanderbilt and R. D. King-Smith, Electric polarization as a bulk quantity and its relation to surface charge, Phys. Rev. B 48, 4442 (1993).

[12] G. van Miert, C. Ortix, and C. M. Smith, Topological origin of edge states in two-dimensional inversion-symmetric insulators and semimetals, 2D Mater. 4, 015023 (2016).

[13] T. L. Hughes, E. Prodan, and B. A. Bernevig, Inversionsymmetric topological insulators, Phys. Rev. B 83, 245132 (2011).

[14] A. M. Turner, Y. Zhang, R. S. K. Mong, and A. Vishwanath, Quantized response and topology of magnetic insulators with inversion symmetry, Phys. Rev. B 85, 165120 (2012).

[15] C. Fang, M. J. Gilbert, and B. A. Bernevig, Bulk topological invariants in noninteracting point group symmetric insulators, Phys. Rev. B 86, 115112 (2012).

[16] A. Alexandradinata, X. Dai, and B. A. Bernevig, Wilson-loop characterization of inversion-symmetric topological insulators, Phys. Rev. B 89, 155114 (2014).

[17] M. Sitte, A. Rosch, E. Altman, and L. Fritz, Topological Insulators in Magnetic Fields: Quantum Hall Effect and Edge Channels with a Nonquantized $\theta$ Term, Phys. Rev. Lett. 108, 126807 (2012).

[18] F. Zhang, C. L. Kane, and E. J. Mele, Surface State Magnetization and Chiral Edge States on Topological Insulators, Phys. Rev. Lett. 110, 046404 (2013).

[19] J. C. Y. Teo and T. L. Hughes, Existence of Majorana-Fermion Bound States on Disclinations and the Classification of Topological Crystalline Superconductors in Two Dimensions, Phys. Rev. Lett. 111, 047006 (2013).

[20] W. A. Benalcazar, J. C. Y. Teo, and T. L. Hughes, Classification of two-dimensional topological crystalline superconductors and Majorana bound states at disclinations, Phys. Rev. B 89, 224503 (2014).

[21] K. Hashimoto, X. Wu, and T. Kimura, Edge states at an intersection of edges of a topological material, Phys. Rev. B 95, 165443 (2017).

[22] W. A. Benalcazar, B. A. Bernevig, and T. L. Hughes, Quantized electric multipole insulators, Science 357, 61 (2017).

[23] C. Fang and L. Fu, New classes of topological crystalline insulators having surface rotation anomaly, Sci. Adv. 5, eaat2374 (2019).

[24] J. Langbehn, Y. Peng, L. Trifunovic, F. von Oppen, and P. W. Brouwer, Reflection-Symmetric Second-Order Topological Insulators and Superconductors, Phys. Rev. Lett. 119, 246401 (2017).

[25] Z. Song, Z. Fang, and C. Fang, $(d-2)$-Dimensional Edge States of Rotation Symmetry Protected Topological States, Phys. Rev. Lett. 119, 246402 (2017).

[26] W. A. Benalcazar, B. A. Bernevig, and T. L. Hughes, Electric multipole moments, topological multipole moment pumping, and chiral hinge states in crystalline insulators, Phys. Rev. B 96, 245115 (2017).

[27] M. Serra-Garcia, V. Peri, R. Süsstrunk, O. R. Bilal, T. Larsen, L. G. Villanueva, and S. D. Huber, Observation of a phononic quadrupole topological insulator, Nature 555, 342 (2018).

[28] M. Ezawa, Higher-Order Topological Insulators and Semimetals on the Breathing Kagome and Pyrochlore Lattices, Phys. Rev. Lett. 120, 026801 (2018).

[29] C. W. Peterson, W. A. Benalcazar, T. L. Hughes, and G. Bahl, A quantized microwave quadrupole insulator with topologically protected corner states, Nature 555, 346 (2018).

[30] M. Ezawa, Magnetic second-order topological insulators and semimetals, Phys. Rev. B 97, 155305 (2018).

[31] M. Ezawa, Strong and weak second-order topological insulators with hexagonal symmetry and $\mathbb{Z}_{3}$ index, Phys. Rev. B 97, 241402(R) (2018).

[32] M. Geier, L. Trifunovic, M. Hoskam, and P. W. Brouwer, Second-order topological insulators and superconductors with an order-two crystalline symmetry, Phys. Rev. B 97, 205135 (2018).

[33] E. Khalaf, Higher-order topological insulators and superconductors protected by inversion symmetry, Phys. Rev. B 97 205136 (2018).

[34] F. Schindler, A. M. Cook, M. G. Vergniory, Z. Wang, S. S. P. Parkin, B. A. Bernevig, and T. Neupert, Higher-order topological insulators, Sci. Adv. 4, eaat0346 (2018).

[35] M. Ezawa, Minimal models for wannier-type higher-order topological insulators and phosphorene, Phys. Rev. B 98, 045125 (2018).

[36] F. Schindler, Z. Wang, M. G. Vergniory, A. M. Cook, A. Murani, S. Sengupta, A. Y. Kasumov, R. Deblock, S. Jeon, I. Drozdov et al., Higher-order topology in bismuth, Nat. Phys. 14, 918 (2018).

[37] G. van Miert and C. Ortix, Higher-order topological insulators protected by inversion and rotoinversion symmetries, Phys. Rev. B 98, 081110(R) (2018).

[38] E. Khalaf, H. C. Po, A. Vishwanath, and H. Watanabe, Symmetry Indicators and Anomalous Surface States of Topological Crystalline Insulators, Phys. Rev. X 8, 031070 (2018).

[39] S. Imhof, C. Berger, F. Bayer, J. Brehm, L. W. Molenkamp, T. Kiessling, F. Schindler, C. H. Lee, M. Greiter, T. Neupert et al., Topolectrical-circuit realization of topological corner modes, Nat. Phys. 14, 925 (2018).

[40] A. Matsugatani and H. Watanabe, Connecting higher-order topological insulators to lower-dimensional topological insulators, Phys. Rev. B 98, 205129 (2018).

[41] B. J. Wieder and B. A. Bernevig, The axion insulator as a pump of fragile topology, arXiv: 1810.02373

[42] S. H. Kooi, G. van Miert, and C. Ortix, Inversion-symmetry protected chiral hinge states in stacks of doped quantum Hall layers, Phys. Rev. B 98, 245102 (2018).

[43] L. Trifunovic and P. W. Brouwer, Higher-Order Bulk-Boundary Correspondence for Topological Crystalline Phases, Phys. Rev. X 9, 011012 (2019).

[44] J. Ahn and B.-J. Yang, Symmetry representation approach to topological invariants in $C_{2 z} T$-symmetric systems, Phys. Rev. B 99, 235125 (2019). 
[45] H. Araki, T. Mizoguchi, and Y. Hatsugai, $\mathbb{Z}_{Q}$ berry phase for higher-order symmetry-protected topological phases, Phys. Rev. Research 2, 012009 (2020).

[46] Y. Hwang, J. Ahn, and B.-J. Yang, Fragile topology protected by inversion symmetry: Diagnosis, bulk-boundary correspondence, and wilson loop, Phys. Rev. B 100, 205126 (2019).

[47] R. Okugawa, S. Hayashi, and T. Nakanishi, Second-order topological phases protected by chiral symmetry, Phys. Rev. B 100, 235302 (2019).

[48] J. C. Y. Teo, L. Fu, and C. L. Kane, Surface states and topological invariants in three-dimensional topological insulators: Application to $\mathrm{Bi}_{1-x} \mathrm{Sb}_{x}$, Phys. Rev. B 78, 045426 (2008).

[49] Y. Tanaka, R. Takahashi, and S. Murakami, Appearance of hinge states in second-order topological insulators via the cutting procedure, Phys. Rev. B 101, 115120 (2020)..

[50] H. C. Po, A. Vishwanath, and H. Watanabe, Symmetry-based indicators of band topology in the 230 space groups, Nat. Commun. 8, 50 (2017).

[51] B. Bradlyn, L. Elcoro, J. Cano, M. G. Vergniory, Z. Wang, C. Felser, M. I. Aroyo, and B. A. Bernevig, Topological quantum chemistry, Nature 547, 298 (2017).

[52] S. Ono and H. Watanabe, Unified understanding of symmetry indicators for all internal symmetry classes, Phys. Rev. B 98, 115150 (2018).
[53] X.-L. Qi, T. L. Hughes, and S.-C. Zhang, Topological field theory of time-reversal invariant insulators, Phys. Rev. B 78, 195424 (2008).

[54] L. Fu and C. L. Kane, Topological insulators with inversion symmetry, Phys. Rev. B 76, 045302 (2007).

[55] J.-W. Rhim, J. Behrends, and J. H. Bardarson, Bulk-boundary correspondence from the intercellular Zak phase, Phys. Rev. B 95, 035421 (2017).

[56] G. van Miert and C. Ortix, Excess charges as a probe of onedimensional topological crystalline insulating phases, Phys. Rev. B 96, 235130 (2017).

[57] F. de Juan, A. Rüegg, and D.-H. Lee, Bulk-defect correspondence in particle-hole symmetric insulators and semimetals, Phys. Rev. B 89, 161117(R) (2014).

[58] H. Watanabe and M. Oshikawa, Inequivalent Berry Phases for the Bulk Polarization, Phys. Rev. X 8, 021065 (2018).

[59] D. Zhang, M. Shi, T. Zhu, D. Xing, H. Zhang, and J. Wang, Topological Axion States in the Magnetic Insulator $\mathrm{MnBi}_{2} \mathrm{Te}_{4}$ with the Quantized Magnetoelectric Effect, Phys. Rev. Lett. 122, 206401 (2019).

[60] J. Li, Y. Li, S. Du, Z. Wang, B.-L. Gu, S.-C. Zhang, K. He, W. Duan, and $\mathrm{Y} . \mathrm{Xu}$, Intrinsic magnetic topological insulators in Van der Waals layered $\mathrm{MnBi}_{2} \mathrm{Te}_{4}$-family materials, Sci. Adv. 5, eaaw5685 (2019). 